\title{
Tracing Outflowing Metals in Simulations of Dwarf and Spiral Galaxies
}

\author{
Charlotte R. Christensen ${ }^{1}$ (i), Romeel Davé ${ }^{2}$ (1), Alyson Brooks ${ }^{3}$ (1) Thomas Quinn $^{4}$, and Sijing Shen ${ }^{5}$ (1) \\ ${ }^{1}$ Physics Department, Grinnell College, 1116 Eighth Avenue, Grinnell, IA 50112, USA; christenc@ grinnell.edu \\ ${ }^{2}$ School of Physics and Astronomy, The University of Edinburgh, Royal Observatory Edinburgh, Blackford Hill, Edinburgh, EH9 3HJ, UK \\ ${ }^{3}$ Department of Physics and Astronomy, Rutgers University, the State University of New Jersey, 136 Frelinghuysen Road, Piscataway, NJ 08854-8019, USA \\ ${ }_{5}^{4}$ Astronomy Department, University of Washington, 3910 15th Avenue NE, Seattle, WA 98195-0002, USA \\ ${ }^{5}$ Institute of Theoretical Astrophysics, University of Oslo, Postboks 1029, Blindern, NO-0315 Oslo, Norway \\ Received 2018 March 26; revised 2018 August 22; accepted 2018 September 14; published 2018 November 9
}

\begin{abstract}
We analyze the metal accumulation in dwarf and spiral galaxies by following the history of metal enrichment and outflows in a suite of 20 high-resolution simulated galaxies. These simulations agree with the observed stellar and gas-phase mass-metallicity relation, an agreement that relies on large fractions of the produced metals escaping into the circumgalactic media. For instance, in galaxies with $M_{\text {vir }} \sim 10^{9.5}-10^{10} M_{\odot}$, we find that about $\sim 85 \%$ of the available metals are outside of the galactic disk at $z=0$, although the fraction decreases to a little less than half in Milky-Way-mass galaxies. In many cases, these metals are spread far beyond the virial radius. We analyze the metal deficit within the ISM and stars in the context of previous work tracking the inflow and outflow of baryons. Outflows are prevalent across the entire mass range, as is reaccretion. We find that between $40 \%$ and $80 \%$ of all metals removed from the galactic disk are later reaccreted. The outflows themselves are metal-enriched relative to the ISM by a factor of 0.2 dex because of the correspondence between sites of metal enrichment and outflows. As a result, the metal mass loading factor scales as $\eta_{\text {metals }} \propto v_{\text {circ }}^{-0.91}$, a somewhat shallower scaling than the total mass loading factor. We analyze the simulated galaxies within the context of analytic chemical evolution models by determining their net metal expulsion efficiencies, which encapsulate the rates of metal loss and reaccretion. We discuss these results in light of the inflow and outflow properties necessary for reproducing the mass-metallicity relation.
\end{abstract}

Key words: galaxies: abundances - galaxies: evolution - intergalactic medium - methods: numerical

\section{Introduction}

Galaxies evolve through a balance between gas accretion and outflows. Cosmological accretion of gas from the intergalactic media enables the continued growth of halos (Nelson et al. 2013), and metal-poor cold-gas accretion has been detected through absorption on the outskirts of galaxies (Kacprzak et al. 2012; Bouche et al. 2013; Crighton et al. 2013). Additionally, reaccretion of previously ejected material provides continuous fuel and can easily dominate over cosmological accretion in galaxies with halo masses $>10^{11}-10^{12} M_{\odot}$ (Oppenheimer et al. 2010). This material exists as metal-enriched gas in the circumgalactic media (CGM) prior to its reaccretion (Cheung et al. 2016).

Meanwhile, gas loss from galaxies is accomplished through feedback-driven outflows. Such outflows are ubiquitous in high-redshift star-forming galaxies and local starburst galaxies (e.g., Heckman et al. 1990; Pettini et al. 2001; Shapley et al. 2003; Martin 2005; Weiner et al. 2009; Steidel et al. 2010; Rubin et al. 2014), and both semi-analytic models and simulations have found them to be necessary to reproduce key observations such as the stellar mass-halo mass relation (Scannapieco et al. 2012; Stinson et al. 2012b; Henriques et al. 2013; Hopkins et al. 2013; White et al. 2015). Together with accretion, outflows set the baryonic content within the disk and regulate star formation (Davé et al. 2012; Lilly et al. 2013; Dekel \& Mandelker 2014; Christensen et al. 2016).

In addition to regulating the baryonic content in galaxies, outflows are key to establishing their metal content. For example, comparisons of the total metals within the interstellar media and stellar disk to the total mass of metals produced predict that $20 \%-25 \%$ of metals remain in the stars and ISM of
Milky-Way-mass galaxies (Peeples et al. 2014) and 6\% remained within the stars and ISM of a dwarf galaxy (McQuinn et al. 2015). As a result, outflows are a leading candidate to regulate the metallicity within the disks of galaxies and establish the mass-metallicity relation (MZR; e.g., Tremonti et al. 2004; Finlator \& Davé 2008; Ma et al. 2016) and its second-parameter dependences on star formation rate and gas content (Davé et al. 2012). The amplitude and slope of the MZR can be explained by the tendency of outflows to reduce the effective yield and by the greater efficiency of outflows in removing material from low-mass halos in combination with their reduced star formation efficiency. While analytic models can explain the MZR by parameterizing metal inflow and outflow efficiencies (Erb 2008; Spitoni et al. 2010; Peeples \& Shankar 2011; Davé et al. 2012; Lilly et al. 2013), these models generally do not account for the reaccretion of metal-enriched material. Additionally, many of these models assume that outflows share the same metallicity as the ISM, while observations show evidence for metal enrichment compared to the ISM (Chisholm et al. 2016). Understanding the rates of reaccretion and the relative enrichment of outflows is key to understanding the source of the MZR.

A corollary to the outflow-driven metal depletion of disk material is the redistribution of metals to the CGM and beyond. Since metals originate primarily in the stellar disks of galaxies, their presence throughout the CGM provides a tracer of the history of inflows and outflows. In particular, strong transport of metals by galactic outflows is indicated by the large, oxygenrich halos surrounding present-day (Prochaska et al. 2011; Tumlinson et al. 2011) and high-redshift (Lehner et al. 2014) star-forming galaxies. Observations of metal-line absorption 
around dwarf galaxies (Bordoloi et al. 2014), around the Andromeda galaxy (Lehner et al. 2015), and throughout the intergalactic medium (IGM; e.g., Cooksey et al. 2013; D'Odorico et al. 2013; Michael Shull et al. 2014) provide additional evidence for outflow-driven enrichment. On the theoretical side, simulations generally require strong outflows from stellar feedback in order to reproduce the rapidly advancing observations of metal lines around galaxies (e.g Stinson et al. 2012b; Ford et al. 2013; Hummels et al. 2013; Shen et al. 2013; Suresh et al. 2015). Metal-line absorption observations also provide a range of constraints to the thermal and dynamical states of the CGM and indicate a primarily bound, multiphase CGM with photoionized and/or collisionally ionized gas embedded in a hotter low-density medium (for a review, see Tumlinson et al. 2017).

Galaxy-formation simulations can both provide the provenance of metals in the disk and halo, and establish the history of metal accretion and outflow. Simulations thus far have primarily focused on examining total baryonic mass loss and reaccretion (e.g., Oppenheimer et al. 2010; Woods et al. 2014; Muratov et al. 2015; Christensen et al. 2016; Anglés-Alcázar et al. 2017). They have tended to converge on mass loading factors with mass scalings between those expected for momentum- and energy-conserving winds. Simulations have also tended to agree that recycling of material is common, fuels late-time star formation (Oppenheimer et al. 2010; Woods et al. 2014), and modifies the angular momentum profile (Brook et al. 2012; Übler et al. 2014; Christensen et al. 2016). However, the fate of outflowing gas, including the rates and timescales of outflow reaccretion, are highly model-dependent, illustrating the importance of largely unexamined processes happening within the CGM. The examination of metals within and surrounding galaxy halos can help delineate between models by, for instance, tracing the eventual distribution of stellar-enriched material. As an example of this type of theoretical investigation, Shen et al. (2012) found satellite progenitors and nearby dwarf galaxies to be the source of $40 \%$ of metals within $3 R_{\mathrm{vir}}$ of a $z=3$ progenitor of a Milky-Waymass halo. In a different investigation, Muratov et al. (2017) found high recycling rates of metals at early times and in lowmass galaxies, leading to similar metallicities of inflowing and outflowing material within the central halos. In contrast, outflows from their $L^{*}$ galaxies at low redshift were very weak, leading to the accumulation of metals within stars. We expand upon these types of studies by following the accumulation of metals within galaxies by tracing gas flows.

Following on the work of Christensen et al. (2016), we use a suite of galaxy-formation simulations to quantify the cycle of metal production, loss, and accretion over two and a half orders of magnitude in virial mass. By tracking the history of smoothed particle hydrodynamic gas particles, we identify instances of accretion and ejection, determine the eventual location of the metals produced by the galaxy, and measure the metallicity of the outflows. In Section 2, we present the suite of simulations and describe the analysis. Results are presented for the redshift zero metal census (Section 3.2) and metal distribution (Section 3.3), the history of metal cycling (Section 3.4), the metallicity of outflows (Section 3.5), and the metal mass loading factor (Section 3.6). These results are discussed in light of the MZR and other works (Section 4), and our conclusions are presented in Section 5.

\section{Simulation and Analysis}

We used cosmological simulations of seven individual volumes to follow the history of metals in 20 field galaxies with final virial masses between $10^{9.5}$ and $10^{12} M_{\odot}$ (Table 1 ). This is the same set of simulations analyzed in Christensen et al. (2016). An overview of the sample is given below, and a description with greater detail can be found in Brooks et al. (2017).

These simulations were computed using the $\mathrm{N}$-body + SPH code, GASOLINE (Wadsley et al. 2004). GASOLINE is an SPH extension to the parallel, gravity-tree-based $N$-body code PKDGRAV (Stadel 2001). The simulations assume a $\Lambda$ CDM cosmology using WMAP3 (Spergel et al. 2007) parameters: $\Omega_{0}=0.24, \Lambda=0.76, h=0.73$, and $\sigma_{8}=0.77$. In order to achieve high resolution while including the cosmological context, we used the "zoom-in" volume renormalization technique (Katz \& White 1993). The final sample includes galaxies selected from a medium-resolution $50^{3} \mathrm{Mpc}^{3}$ volume and a higher resolution $25^{3}$ $\mathrm{Mpc}^{3}$ volume. In the high-resolution (medium-resolution) simulations, the force spline softening length is $\epsilon=87$ (170) pc, and the particle masses for the dark matter, gas, and stars (at their formation) are, respectively, $1.6(13) \times 10^{4}, 3.3(27.0) \times 10^{3}$, and $1.0(8.0) \times 10^{3} M_{\odot}$.

GASOLINE follows non-equilibrium abundances of $\mathrm{H}$ (including $\mathrm{H}_{2}$ ) and $\mathrm{He}$ species. Photoionization and heating rates are based on a redshift-dependent cosmic ultraviolet background ${ }^{6}$ while $\mathrm{H}_{2}$ dissociation is based on the Lyman-Werner radiation produced by nearby star particles (Christensen et al. 2012). H and $\mathrm{He}$ cooling channels include collisional ionization (Abel et al. 1997), $\mathrm{H}_{2}$ collisions, radiative recombination (Black 1981; Verner \& Ferland 1996), photoionization, bremsstrahlung, and line cooling (Cen 1992) Metal-line cooling rates are calculated from CLOUDY (version 07.02; Ferland et al. 1998) models based on the gas temperature, density, metallicity, and cosmic UV background under the assumptions of ionization equilibrium and optically thin gas.

Star formation proceeds stochastically according to

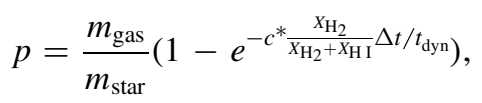

where $p$ is the probability of a gas particle spawning a star particle in a time step $\Delta t, m_{\text {gas }}$ is the mass of the gas particle, $m_{\text {star }}$ is the mass of the potential star, $c^{*}=0.1$ is the starforming efficiency, $X_{\mathrm{H}_{2}}$ and $X_{\mathrm{H} \text { I }}$ are the mass fractions of the particle in the form of $\mathrm{H}_{2}$ and $\mathrm{HI}$, respectively, and $t_{\mathrm{dyn}}$ is the dynamical time. The dependency on the $\mathrm{H}_{2}$ abundance ensures that star formation happens in dense $\left(\rho \gtrsim 10 \mathrm{amu} \mathrm{cm}^{-3}\right)$, cold gas; however, star formation is technically allowed in any gas particle with densities greater than $0.1 \mathrm{amu} \mathrm{cm}^{-3}$ and temperatures less than $10^{3} \mathrm{~K}$.

Energy from Type II supernovae (SNe II) is distributed to the surrounding gas particles according to the "blastwave" approach (Stinson et al. 2006), assuming a Kroupa et al. (1993) initial mass function (IMF) and the canonical $10^{51}$ ergs per SN. In this subgrid recipe, the cooling of feedback-affected particles is disabled for the theoretical lifetime of a hot, low-density shell produced during the momentum-conserving phase of the SN

\footnotetext{
6 The cosmic ultraviolet background used is an unpublished updated version of Haardt \& Madau (1996), specified in CLOUDY (Ferland et al. 1998) as "table HM05."
} 
Table 1

Properties of the Set of Galaxies at $z=0$

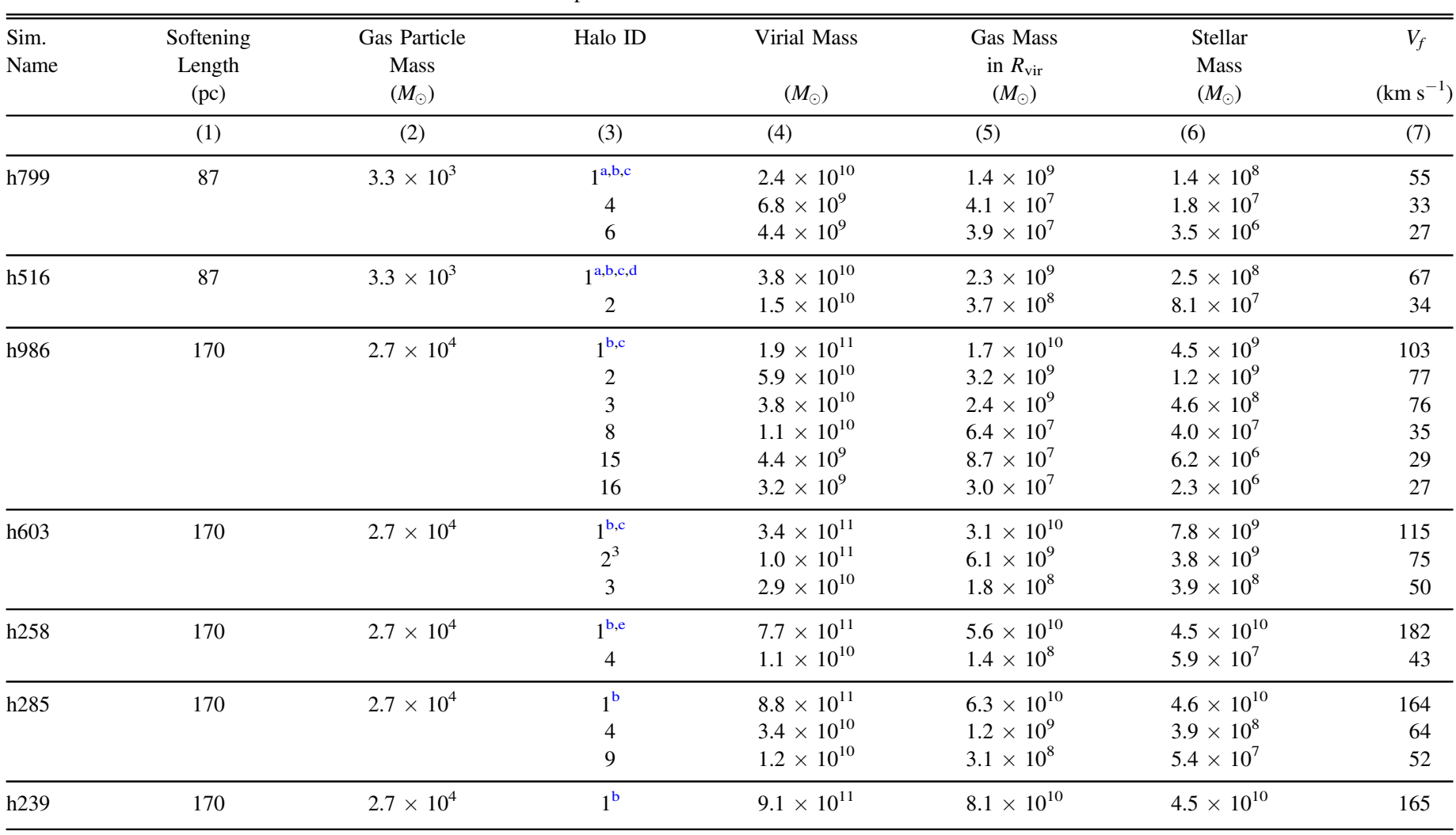

Notes.

a Appears in Governato et al. (2012).

b Appears in Munshi et al. (2013).

c Appears in Christensen et al. (2014).

d Appears in Christensen et al. (2012).

e Appears in Zolotov et al. (2012).

remnant (McKee \& Ostriker 1977). This recipe differs from many other recipes (e.g., Springel \& Hernquist 2003; Davé et al. 2011; Scannapieco et al. 2012) in that no momentum kick is added to the particles; the particle remains hydrodynamically coupled to the rest of the simulation, and the feedback depends only on the local gas properties. We do not include a separate model for other forms of stellar feedback, such as radiation pressure (e.g., Stinson et al. 2012a; Hopkins et al. 2013), that help drive a galactic wind through additional momentum transfer or by making the gas more responsive to the SN feedback. Instead, this blastwave recipe represents the total stellar feedback from young stars.

We follow the production and distribution of oxygen and iron separately. Metals are returned to the ISM both through $\mathrm{SNe}$ I and II and stellar winds. For SNe II, metals are distributed to the same gas particles as the feedback energy, assuming the production rates from Raiteri et al. (1996) with yields from Woosley \& Weaver (1995). SNe Ia are calculated to occur using the rates from Raiteri et al. (1996). Each SN Ia produces $0.63 M_{\odot}$ iron and $0.13 M_{\odot}$ oxygen (Thielemann et al. 1986), which are transferred to the nearest gas particles. Energy from $\mathrm{SNe}$ Ia is also distributed to the gas particles within the smoothing kernel; however, cooling is not disabled for these particles as it is for SNe II. Mass is returned to the ISM by stellar winds using mass loss rates from Weidemann (1987). This mass is distributed to gas particles within the smoothing sphere of the star particle, assuming the same metallicity as the star particle.

Metals are further distributed throughout the gas through diffusion (Shen et al. 2010). In this model, based on Smagorinsky (1963), subgrid turbulent mixing is treated as a shear-dependent diffusion term. As a result, the highest diffusion rates are calculated for shearing flows. Instances of compressive or purely rotating flows result in no diffusion. A tunable parameter, called the metal diffusion coefficient, is used to scale the strength of diffusion. For these simulations, a conservative value of 0.01 was chosen for the metal diffusion coefficient.

\subsection{Postprocessing Analysis}

Individual halos were selected from snapshots of the simulations during postprocessing. We used AMIGA'S HALO FINDER (Gill et al. 2004; Knollmann \& Knebe 2009), ${ }^{7}$ in which areas of overdensity are identified using grid hierarchy and then gravitationally unbound particles are iteratively removed from the prospective halos. The virial radius, $R_{\mathrm{vir}}$, is defined such that the average halo density is a multiple of the background density. This multiple evolves with redshift but is approximately equal to 100 times the critical density at $z=0$. The

\footnotetext{
7 AMIGA'S HALO FINDER is available for download at http://popia.ft.uam. es/AHF/Download.html.
} 
main progenitor of each galaxy was traced back in time from redshift zero through the creation of a merger tree and was defined at each snapshot to be the halo that contained the majority of particles from the subsequent snapshot.

\subsubsection{Inflow/Outflow Identification}

After the main progenitor halo had been identified at each snapshot, we identified all instances of accretion and outflow. To do this, gas particles were classified in each snapshot as being part of the disk, within the halo, and outside the galaxy. Accretion events and ejection were found by identifying the instances when particles moved from one classification to another. The time resolution of this tracking comes from the spacings between snapshots, which were $\sim 100 \mathrm{Myr}$ for all of the simulations. As a result, we were unable to measure accretion and ejection at a time resolution less that $100 \mathrm{Myr}$, and it is possible for accretion and ejection events to have been missed if a particle left and reentered the galaxy between time steps.

We used the following criteria for classifying particles into different components. Gas particles are defined as being in the halo if they are within the virial radius. They are defined as within the disk if they (1) have density $>0.1 \mathrm{amu} \mathrm{cm}^{-3}$, (2) have temperature $<1.2 \times 10^{4} \mathrm{~K}$, and (3) are less than $3 \mathrm{kpc}$ from the plane of the disk. These disk parameters were chosen to thermodynamically select for the ISM and to spatially eliminate cool gas in accreting satellites.

Particles are classified as being accreted onto the halo each snapshot they go from not being part of a halo to being included in it. Similarly, particles are classified as being accreted onto the disk each step they are included in the disk after not having been included in it during the previous snapshot. Particles may, therefore, be accreted onto the disk or halo multiple times. As may be expected, particles are identified as leaving the disk each time they move from disk to halo. However, we also further divided the ejected material between material that is merely heated and material that passes more stringent constraints for ejecta. This division is necessary as it is common for gas particles to be heated by $\mathrm{SNe}$ to temperatures greater than $1.2 \times 10^{4} \mathrm{~K}$ but then to quickly radiate their energy away without having a substantial effect on the disk dynamics. Therefore, we defined the following classifications, with each later classification a subset of the previous ones.

1. Material removed from the disk includes all gas that is classified as disk material in one step and outside of the disk in the next step.

2. Material ejected from the disk includes those particles removed from the disk that become dynamically unbound from the baryonic disk. Specifically, a particle is defined to be "unbound from the disk" at any snapshot after leaving a disk if its velocity exceeds the escape velocity for a mass equal to the sum of the ISM and stellar mass located at the center of the galaxy. This classification is most similar to what is generally referred to as outflows.

3. Material expelled from the halo includes all particles that are classified as being in the disk in one instance and at any later point travel beyond the virial radius. Note that we are focusing our analysis on gas that was ever part of the ISM and so do not include in our analysis gas that was only ever part of the halo prior to leaving it.
Together, these classifications allowed us to determine all gas particles that were ever part of the halo or disk and their history of accretion and outflow.

One additional complication in tracking the flow of metals in and out of galaxies is that metal diffusion is included in the simulation. As a result, metals may pass from within the disk to the halo through diffusion, as opposed to an SPH particle leaving the disk. Therefore, it is best in these circumstances to think of the SPH particles as tracer particles of the metal distribution that pick up on bulk motion while missing some of the small-scale diffusion. Based on comparing the mass of metals within the disk and halo at each time step to that expected from particle tracing, we were able to account for $\gtrsim 90 \%$ of the metal movement from disk to halo and back. Throughout this paper, we note those aspects of the analysis where diffusion could further affect the results and specify how we addressed it.

\section{Results}

\subsection{Mass-Metallicity Relation}

We assess our simulated galaxies with reference to the observed MZR. These simulations were previously shown to be consistent with the redshift zero gas-phase MZR, stellar masshalo mass relation, and the baryonic and $i$-band Tully-Fisher relation (Christensen et al. 2016). Galaxies generated with a previous version of GASOLINE have also been shown to demonstrate an evolving gas-phase MZR (Brooks et al. 2007). Here, we expand our analysis to show our simulated galaxies along the gas-phase MZR at multiple redshifts and the redshift zero stellar MZR.

Figure 1 compares the gas-phase oxygen abundances at $z=3,2.3,0.8$, and 0 to the observed values. In order to best mimic observations, stellar masses are determined from broadband magnitudes using KCORRECT (Blanton \& Roweis 2007). Metallicities were calculated using the star formation rate-weighted average gas particle oxygen abundances. Weighting by the star formation rate (i.e., the probability of the gas particle forming a star) was chosen to mimic the measurement of metallicities in star-forming regions of observed galaxies. The simulated galaxies show a slight increase in the normalization of the MZR with decreasing redshift.

We compare our simulated galaxies to observational data from $z \sim 3$ (Mannucci et al. 2009), $z \sim 2.3$ (Erb et al. 2006; Steidel et al. 2014; Sanders et al. 2015), $z \sim 0.8$ (Zahid et al. 2011), and $z \sim 0$ (Tremonti et al. 2004; Lee et al. 2006; Andrews \& Martini 2013). These observations were made based on different diagnostics using different calibrations, and the systematic uncertainty between the different metallicity diagnostics could be as much as 0.7 dex (Kewley \& Ellison 2008). In order to demonstrate the evolution of the MZR, we showed or converted to the N2 calibration (Pettini \& Pagel 2004) where possible; in particular, we used the formula in Kewley \& Ellison (2008) to transform Tremonti et al. (2004) and Zahid et al. (2011) to the N2 calibration. Compared to observations, the simulations at $z=3$ may have slightly too high of metallicities. However, given the lack of overlap in the mass range and the large systematic uncertainties in the observed metallicities, the simulations appear largely consistent with observations. 

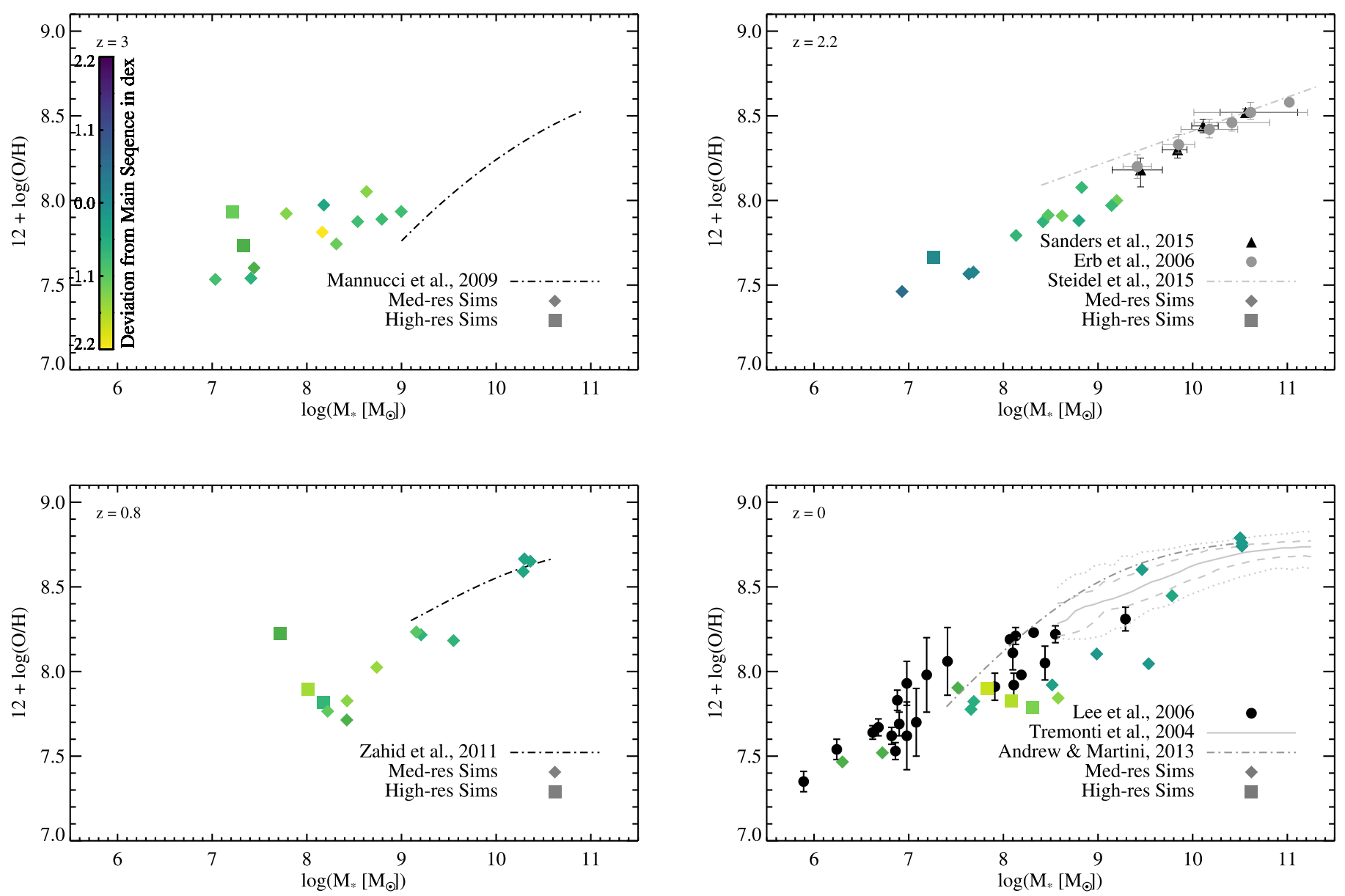

Figure 1. Evolving MZR for simulated galaxies (colored symbols) at redshifts 3.0, 2.3, 0.8, and 0 compared to observations (grayscale lines and symbols). Stellar masses of simulated galaxies were determined from mock photometric observations. The higher resolution simulations are represented by squares and the lower resolution ones by diamonds. The top-left panel compares the redshift 3.0 simulations to the observed sample of Lyman-break galaxies from Mannucci et al. (2009, black dotted-dashed line). The top-right panel compares the redshift 2.3 simulations to data from Erb et al. (2006, gray circles), Steidel et al. (2014, gray dotteddashed line), and Sanders et al. (2015, black triangles) based on N2 calibrations (Pettini \& Pagel 2004). The bottom-left panel compares the redshift 0.8 simulations to data from Zahid et al. (2011, black dotted-dashed line), adjusted to N2 calibration following Kewley \& Ellison (2008). The bottom-right panel compares the redshift zero simulations to data from Tremonti et al. (2004), adjusted to N2 calibration (gray lines, solid showing the median values, dashed enclosing 68\% of the galaxies, and dotted enclosing 95\% of the galaxies). Additional redshift zero observations are shown from Andrews \& Martini (2013, gray dotted-dashed line) and Lee et al. (2006, black circles). Colors represent the amount by which galaxies deviate from the galaxy main sequence, measured in dex. Fits to the observed main sequence for each of the redshift ranges are taken from Salim et al. (2007, $z=0$ ), Whitaker et al. (2014, $z=0.8$ and 2.2), and Santini et al. (2017, $z=3$ ).

We looked for evidence of a second-parameter dependence of the metallicity on star formation rate at a given stellar mass in our sample by coloring the galaxies in Figure 1 by their deviation from the star formation rate expected for their stellar mass. Specifically, the difference between the $\log$ measured specific star formation rate and log expected star formation rate is encoded into color on a scale spanning \pm 2 dex. Star formation rates were calculated from the simulations by averaging over the previous million years and were compared to the star formation rates expected from a fit to the specific star formation rate-stellar mass main sequence at the relevant redshift: Salim et al. (2007) at $z=0$, Whitaker et al. (2014) at $z=0.8$ and 2.2, and Santini et al. (2017) at $z=3$. It is important to recognize, though, that data for galaxies with stellar masses below $10^{9} M_{\odot}$ is rare beyond the local universe so the fits to observational data had to be extrapolated to the relevant stellar mass regime. Our simulations do not show clear evidence for a second-parameter dependence at any redshift. This is likely the result of our small sample size, but also may owe to the fact that most of the galaxies are in the mass regime where the star formation is bursty (as discussed in
Faucher-Giguère 2018), and such burstiness will hide this second-parameter dependence on short timescales (Torrey et al. 2018).

Figure 2 shows the simulated and observed $z=0$ MZR for stellar metallicities. As for the gas-phase MZR, stellar masses are calculated using KCORRECT. In order to better compare with observations, stellar metallicities are weighted by the $K$-band luminosity of the star particle. However, this weighting introduces almost no change to the average metallicity. The simulations are compared to observational data from Gallazzi et al. (2005) and Kirby et al. (2013). We find that the simulations are consistent with observations over 4.5 orders of magnitude, indicating that appropriate masses of metals are retained within the stars, in addition to the ISM. We further compare the fraction of metals present in different phases of the galaxy in the following section.

\subsection{Metal Census}

Observed galaxies show a substantial deficit in the metals contained within their disks (including both stars and the atomic and molecular components of their ISM). For example, the isolated dwarf galaxy Leo P was found to contain only 5\% 


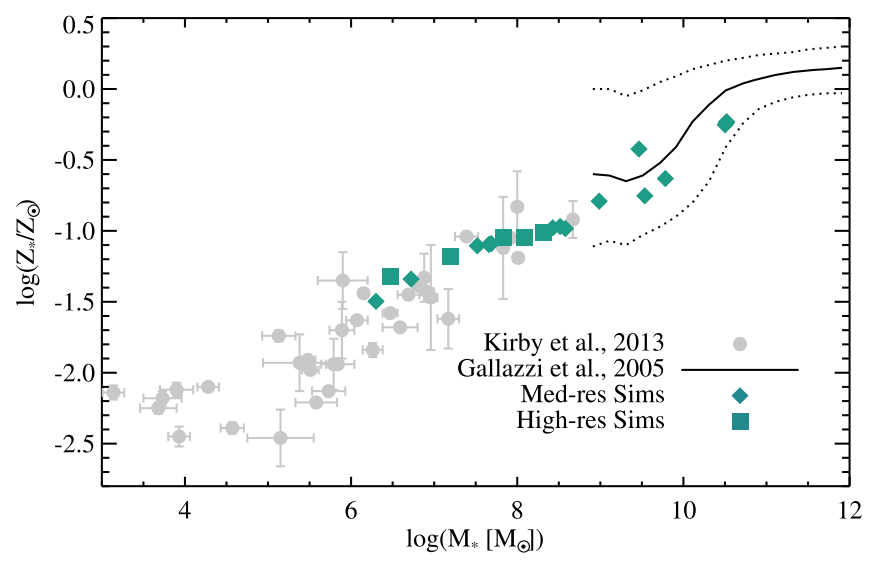

Figure 2. Stellar MZR for simulated galaxies (colored symbols) compared to observed values (grayscale symbols). Redshift zero stellar metallicities from simulations were calculated using the $K$-band weighted average metallicity of all star particles in the galaxy. Stellar masses of simulated galaxies were determined from mock photometric observations. The higher resolution simulations are represented by teal filled squares and the lower resolution ones by diamonds. Gray filled circles represent observational data for dwarf irregular and dwarf spheroidal galaxies from Kirby et al. (2013). Black lines show observational data from Gallazzi et al. (2005); the solid line shows the median values, and the dotted lines show the 16th and 84th percentile data. The simulated galaxies appear consistent with the observed data, and there is no distinction between the two different resolutions.

of all the metals produced by its stars in its disk (1\% in the form of stars; McQuinn et al. 2015). On the more massive side, Peeples et al. (2014) found that in galaxies with stellar masses between $10^{9.2} M_{\odot}$ and $10^{11.6} M_{\odot}$, approximately $20 \%-25 \%$ (with an uncertainty range between $10 \%$ and $40 \%$ ) of the metals produced by the stars remained in the disks of the galaxies. This metal deficit is a companion to the "Missing Baryon Problem"; like the missing baryons, these metals are presumed to largely be contained within the CGM. Here we examine the metal census for our population of galaxies. Since we have excluded satellites (as defined by Amiga Halo Finder) from our sample, our analysis focuses on metals lost for reasons other than stripping by a more massive halo.

Figure 3 shows the fraction of metals available to each galaxy contained within the halo $\left(r<R_{\mathrm{vir}}\right)$, the stars, and the ISM at redshifts two and zero. The mass of metals available is defined to be the sum of all metals produced by the stars within the final halo. To do this, we used the same metal production models included within GASOLINE for SNe II and SNe Ia to calculate the mass of oxygen and iron produced by all star particles given their age and metallicity and assuming a Kroupa et al. (1993) IMF. Since observations do not tally the metals contained within stellar remnants, we also did not include them when calculating the mass of metals contained within the stars. Specifically, we reduced the mass of metals in each star particle by the mass fraction of that particle in the form of stellar remnants based on its age and metallicity.

We find that a substantial fraction of metals are lost from galaxies of all masses; by $z=0$ between $35 \%$ and $85 \%$ of the metals had been removed from the galactic disk and between $15 \%$ and $75 \%$ from the entire halo, as defined by the virial radius. The metal fractions contained in stars show a strong mass dependency with higher mass galaxies retaining a greater fraction of metals in stars. By $z=0$, the fraction of metals contained within the entire halo also shows some evidence for mass scaling for those halos with $M_{*}>10^{7} M_{\odot}$. Within this mass range, lower mass galaxies appear more able to remove
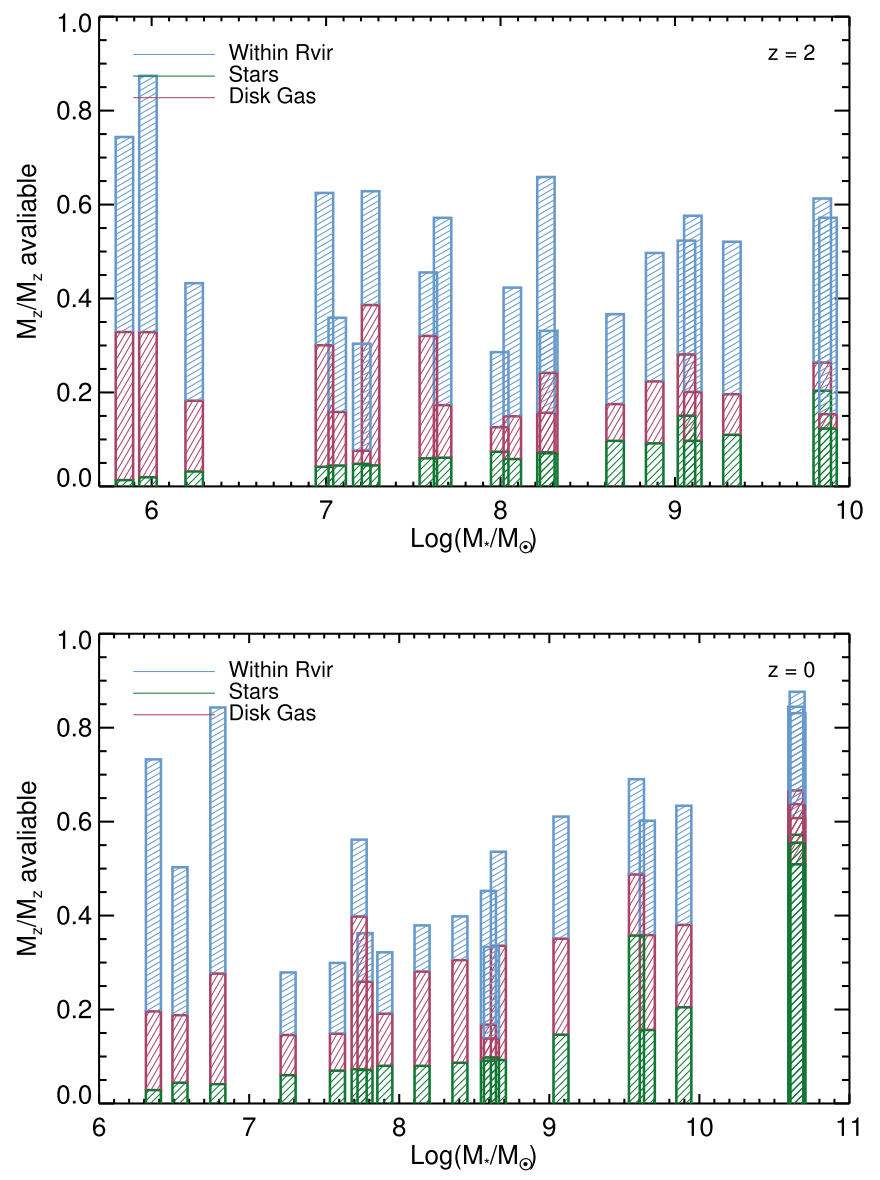

Figure 3. Redshift $z=2$ and $z=0$ location of all the metals produced by each of the galaxies. The metal mass contained in each component is normalized by

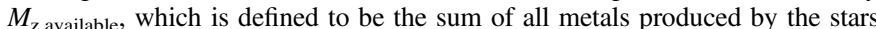
within the final halo. Each bar represents a unique galaxy with the fraction contained within stars shown in green, the fraction contained within the ISM shown in maroon, and the fraction within the virial radius but not within the ISM and stars (i.e., the CGM) shown in blue. In part because of their larger stellar mass, more massive galaxies retain a greater fraction of their metals in stars. The metal fraction retained within the CGM does not show a clear mass trend and, by $z=0$, neither does the fraction retained within the ISM.

metals through outflows, likely because of their lower gravitational potential. This result mirrors a similar one in Christensen et al. (2016), where $20 \%$ of baryons ever accreted to the galaxy were retained within it at $z=0$ for dwarf galaxies, while closer to $80 \%$ were retained within Milky-Waymass galaxies. However, the three galaxies with $z=0$ stellar masses $<10^{7} M_{\odot}$ complicate this mass trend by retaining relatively large fractions of their metals within $R_{\text {vir. These }}$ galaxies may illustrate a transition to a mass range where the low gravitational potentials that could aid metal loss are counterbalanced by incredibly low rates of star formation. Notably, similar fractions of metals are retained within the ISM for galaxies of all masses. Similarly, the fraction of metals retained within the CGM does not show a clear mass trend.

The metal deficit is well established even by $z=2$, with generally $\sim 60 \%$ or less of metals retained within the virial radius. This result is consistent with observations indicating that the missing metals problem is already in place by $z \sim 2$ (e.g., Pagel 1999). In the evolution from $z=2$ to $z=0$, the fraction of metals retained within the lowest mass galaxies $\left(M_{*}<10^{7.5}\right)$ is reduced as outflows continue to expel metals. 
By contrast, the metal mass fraction within the highest mass galaxies increases, primarily as those metals become locked into stars. Across the entire range of galaxies, the fraction of metals within the halo gas tends to decrease over time.

A similar analysis of numerical simulations in Muratov et al. (2017) found comparable $z=0$ mass trends in metal mass loss. As in ours, they found that greater fractions of the available metals were locked within stars for Milky-Way-mass galaxies than for dwarf galaxies, while the fraction retained within the ISM showed a negligible mass trend. However, higher amounts of metals were retained in the CGM, stars, and ISM in their simulations than in ours. The difference was greatest in the Milky-Way-mass galaxies $\left(M_{*} \sim 4 \times 10^{10} M_{\odot}\right)$. While we find that $50 \%-60 \%$ of available metals are retained in stars and $80 \%-90 \%$ within a virial radius at a redshift of zero, they found closer to $80 \%$ in stars and $>90 \%$ within a virial radius. Differences between these results are most likely due to differences in implementing feedback, as will be discussed further in Section 4.

Observational constraints for halos in this mass range are limited. Nevertheless, we draw some comparisons at both the high and the low ends. The largest survey of the fraction of metals retained in stars within dwarf galaxies are for eight dwarf spheroidal Milky Way satellites with stellar masses between $5.6 \times 10^{5}$ and $1.8 \times 10^{7} M_{\odot}$ Kirby et al. (2011). They determined that $<1 \%-4 \%$ of the metals produced by the stars in their sample of galaxies were retained within the stellar component (with the greatest fraction retained in the most massive dwarf), which they found suggestive of an energydriven scaling for the mass loading factor. In comparison, we found that our four galaxies in this mass range retained a similar fraction of their metals in stars (specifically, 3\%, 4\%, $4 \%$, and $6 \%$ for the galaxies with masses between $2.3 \times 10^{6}$ and $\left.1.8 \times 10^{7} M_{\odot}\right)$. Likewise, these simulations exhibit mass loading factors with energy-driven scalings (Christensen et al. 2016). Despite this apparent agreement, it is dangerous to draw strong conclusions from this comparison, as the observed sample of dwarf spheroidal galaxies has a substantially different environment and evolutionary history than our field dwarf irregular galaxies. In particular, tidal and/or ram pressure stripping acting on the observed satellites may have impacted the fraction of metals retained within stars by contributing to metal loss prior to the cessation of star formation. Furthermore, we cannot compare the fraction of metals retained within the ISM for our sample to data from Kirby et al. (2011), as dwarf spheroidal galaxies are necessarily lacking an ISM because of their satellite environment.

Observational measurements of the metal census for field dwarf galaxies are difficult to achieve because $\mathrm{H}$ II regions are required in addition to stellar spectroscopy. The only currently available observational metal census for a field dwarf galaxy is for Leo P (McQuinn et al. 2015). Leo P shows no evidence of interaction and has a metallicity consistent with a lowluminosity extension of the MZR, implying that it is a representative galaxy. It has a stellar mass half that of our lowest mass galaxy and has a similar, although slightly smaller, metal fraction retained within its stars as our two lowest mass galaxies $(3 \%$ and $4 \%$ for the simulated dwarf galaxies and $\sim 1 \%$ in Leo P; McQuinn et al. 2015). When comparing the fraction of metals retained within the ISM, it is important to use similar definitions in selecting ISM material. In Figure 3, we use the same definition of "disk" material as in our particle tracing code: (1) density $>0.1 \mathrm{amu} \mathrm{cm}^{-3}$, (2) temperature $<1.2 \times 10^{4} \mathrm{~K}$, and (3) less than $3 \mathrm{kpc}$ from the plane of the disk (Section 2.1.1). However, observations generally (and in the case of Leo P, specifically) measure ISM mass through H I and, when detectable, $\mathrm{H}_{2}$. In low-mass halos, in particular, the difference between these two ISM definitions can be significant. Therefore, we also calculate the fraction of metals retained within the ISM as determined by scaling gas particles within $3 \mathrm{kpc}$ of the disk plane by their $\mathrm{HI}$ and $\mathrm{H}_{2}$ content. We calculate that our two lowest mass galaxies retained $9 \%$ and $10 \%$ of their metals within the ISM defined this way, compared to the $\sim 4 \%$ determined for Leo P. This factor of 2 difference may imply that the simulations retain too many of their metals in their ISM. Or it is possible that the discrepancy can be explained by the differences in stellar masses between Leo $\mathrm{P}$ and the simulations, and the stochasticity in metal retention among dwarf galaxies. Larger samples of both observed and simulated dwarf galaxies will be needed to draw firmer conclusions as to the consistency of the results.

For our most massive galaxies, the metals retained can be compared with observations from Peeples et al. (2014). The average $64 \%$ of metals we found retained in Milky-Way-mass galaxy disks is substantially greater than the $\sim 25 \%$ measured by Peeples et al. (2014). This is a qualitatively similar but smaller level of disagreement than Muratov et al. (2017) had with Peeples et al. (2014). It is possible that this discrepancy could argue for the need for an additional form of feedback, such as AGN feedback, in the most massive of our galaxies. However, comparisons to the gas and stellar MZR confirm that the metallicities in our simulations are consistent with observed values (Section 3.1). Given that the metal content within the ISM and stars agrees with observations, the discrepancy with Peeples et al. (2014) almost certainly originates from differences in how the available metal mass is calculated. While GASOLINE uses the yields from Woosley \& Weaver (1995) for SNe II, Peeples et al. (2014) assumed higher yields based on other models. Additionally, Peeples et al. (2014) assumed significantly higher mass loss rates $(\sim 55 \%)$ from simple stellar populations using a Chabrier (2003) IMF than GASOLINE does using a Kroupa et al. (1993) IMF. As a result, Peeples et al. (2014) calculated about four times as much metal mass available for the same stellar mass as our simulations produce. Assuming a higher value of $M_{z \text {,available than what is }}$ actually used in the simulations profoundly reduces the presumed fraction of metals retained in both the disk and the CGM (defined to be material within $150 \mathrm{kpc}$ of the galaxy in Peeples et al. 2014).

By changing our calculation of the metals available (and, to a much lesser extent, how we select for ISM and CGM material) to be consistent with Peeples et al. (2014), we can compare Milky-Way-mass galaxies $\left(\log \left(M_{*} / M_{\odot}\right) \sim 10.6\right)$ and slightly lower mass spiral galaxies $\left(9.5<\log \left(M_{*} / M_{\odot}\right)<10.0\right)$ to measurements from Peeples et al. (2014). Specifically, under these assumptions, the simulated Milky-Way-mass galaxies are predicted retain on average $13 \%$ of their metals in their disk and $17 \%$ within $150 \mathrm{kpc}$, compared to the observed $\sim 25 \%$ and $>30 \%$ for those components. Similarly, the simulated lower mass spiral galaxies would be predicted to retain on average $8 \%$ in their disk and $15 \%$ within $150 \mathrm{kpc}$, compared to the observed $\sim 20 \%$ and $>40 \%$. So, by changing the calculation of $M_{z \text {,available }}$ to follow the method in Peeples et al. (2014), we moved from predicting about twice as many metals retained in 

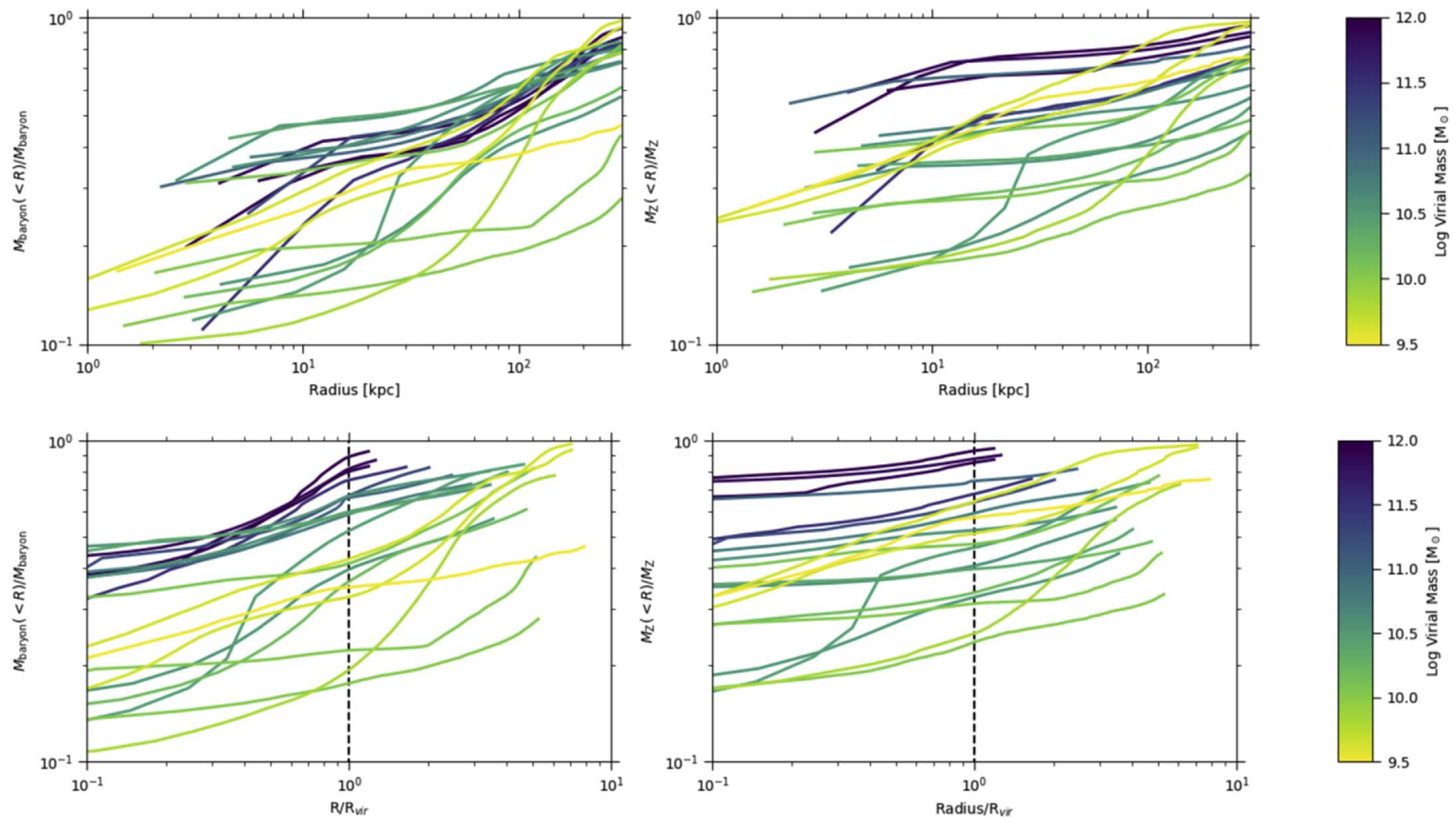

Figure 4. Normalized, cumulative histograms of the $z=0$ location of the mass (left) and metals (right) ever within the galaxy halo since $z=3$ as a function of radius. The top panels show the absolute distances, while in the bottom panels the distances are scaled by the virial radius of the corresponding galaxy. The line colors, spanning from yellow (low mass) to purple (high mass), represent the galaxy mass. The shape of the curves and the relationship between different galaxies are similar for the mass and metal histograms. However, the metal mass fraction tends to be higher at small radii and flatten off more slowly at large radii.

the CGM and disks of spiral galaxies to half as many. Therefore, we cannot yet claim agreement with Peeples et al. (2014), but uncertainties in metal yields also limit the ability of observations to constrain the simulations.

\subsection{Redshift Zero Distribution of Metals}

Observations of the CGM have shown metals distributed out to the virial radius (e.g., Tumlinson et al. 2011), and in some cases, O VI has been observed out to $5 R_{\text {vir }}$ (Pratt et al. 2018), demonstrating the far reach of outflows. Simulations have also highlighted the reach of outflows, especially in low-mass galaxies. Ma et al. (2016) found that their dwarf galaxies retained only $2 \%-20 \%$ of their metals within a virial radius, while Shen et al. (2014) calculated that $87 \%$ of the metals produced by a group of seven dwarf galaxies were spread over a $3^{3} \mathrm{Mpc}^{3}$ volume, equating to a distance of $\sim 17.5 R_{\mathrm{vir}}$ of the most massive dwarf galaxy. Here, we examine the extent of metal enrichment of the CGM by showing the metal fraction contained within a given radius. In Figure 4, the left-hand panels shows the normalized cumulative histogram of the $z=0$ locations of gas or stars ever part of the galaxy halo since the start of the simulation. The right-hand panels weight those particles by their metal content to demonstrate the eventual distribution of metals. The top panels show the physical distribution of the matter, while the bottom panels show the distances scaled by the virial radius. All histograms are shown out to $300 \mathrm{kpc}$. This distance corresponds to the largest impact parameter typically used for observations of the CGM and ensures that the analysis is within the highest resolved regions of the simulation. Note that the normalization factor for this plot differs from the mass of metals available used in Section 3.2, as in this plot only those metals contained within gas or star particles that were ever part of the main progenitor are considered.

Since metal diffusion can occur across gas particles, it is possible that the eventual location of some metals may not be the same as the gas particle they exited the halo with. More exactly, one may consider the gas particles to be tracer particles associated with the underlying metal distribution. Therefore, the eventual location of the gas particles follows the bulk motion of the metals at a limited resolution. In order to avoid underestimating the mass of metals exiting the virial radius because of metal diffusion we make the following adjustment when generating Figure 4. For those particles that exit the virial radius, we consider their metallicity at the time they exit, while in all other instances the redshift zero metallicity is used.

As would be expected from the substantial fractions of metals beyond the virial radius (Figure 3), the metal enrichment continues far beyond $R_{\mathrm{vir}}$. The distribution of both total mass and metals relative to the virial radii shows clear trends with galaxy mass. Metals and total mass tend to remain closer to the centers of more massive galaxies because of their larger gravitational potential. However, this mass trend is complicated by the three lowest mass galaxies, whose metals are less dispersed than most of the medium-mass galaxies. The lack of mass and metal dispersal in these smallest galaxies likely results from their extremely low star formation rates.

We find that on average about $78 \%$ of metals are contained within the virial radii of the three most massive halos. Galaxies with virial masses between $10^{9.5}$ and $10^{10.5} M_{\odot}$ only retain on average $45 \%$ percent of their metals within a virial radius. In 


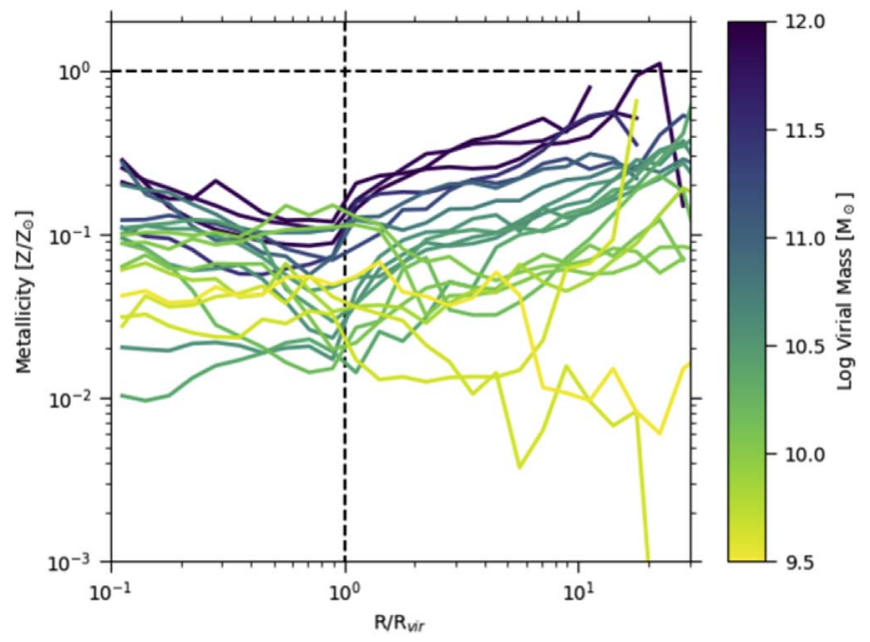

Figure 5. Metallicity of the material ever once within $R_{\text {vir }}$, calculated from the ratio of the histogram of metals to the histogram of the mass. The spatial distributions are scaled by the virial radius of each of the galaxies. As in Figure 4, the line colors, spanning from yellow (low mass) to purple (high mass), represent galaxy mass. Metallicities generally decrease out to $R_{\text {vir }}$. Only those particles ever within $R_{\text {vir }}$ are included in this analysis, so particles beyond $R_{\text {vir }}$ at $z=0$ were likely once part of an outflow. This selection explains the frequent rise in metallicity beyond $R_{\mathrm{vir}}$.

general, the trend of increasing dispersal relative to the virial radius with decreasing virial mass is similar to that found by Ma et al. (2016), although these simulations have slightly more metals retained within a virial radius for dwarfs and slightly fewer for Milky-Way-mass galaxies than in Ma et al. (2016). For instance, in Ma et al. (2016), essentially all metals produced by their Milky-Way-mass galaxy were retained within one virial radius (and almost all within $0.1 R_{\text {vir }}$ ) while their $M_{\text {vir }}=2.5 \times 10^{9} M_{\odot}$ galaxy retained only $2 \%$ of its metals within a virial radius.

Metals are generally more likely to be retained close to the center of the galaxy than total mass. This phenomenon is apparent in the shallowness of the histograms of cumulative metals at very small radii. It can be seen even more clearly through the average metallicity of the particles-i.e., the ratio of the histogram of metals to the histogram of the mass that was ever within the galaxy disk (Figure 5). For almost all of the galaxies, the metallicity is relatively high at the center and drops toward the virial radius. This relatively high metal retention can be explained by the tendency of star formation to occur throughout the galactic disk, where the gas is comparatively metal-enriched, resulting in metals becoming locked into stars. After the virial radius, the metallicities tend to rise again. The change from inside to outside of the virial radius is a result of our selection-since only those particles that were once within the virial radius are analyzed, those particles that are outside of the virial radius at $z=0$ are especially likely to have been part of an outflow. The frequent continued rise in metallicity after $R_{\text {vir }}$ results from the correlation between metal injection and SN energy. Those particles that travel far distances most likely received large amounts of both energy and metals.

\subsection{History of Metal Enrichment}

The history of metal enrichment of both the CGM and ISM can be seen by chronicling pristine accretion, star formation, outflows, and reaccretion. At any point in time, the metal content within the ISM and stars is the sum of the metals accreted from outside the halo (either in the form of gas or stars) and the metals produced by the stars within the galaxy minus the net metal loss in outflows. This net metal loss is the total mass of metals that have left the galaxy minus the mass of metals that reaccreted onto it. In Figure 6, we show the history of these processes and the total metal mass contained within the ISM and stars as a function of time for each of the simulated galaxies.

Metal production within the galaxy by stars is shown by the long-dashed green line. Similar to the amount of "metals available" generated for Figure 3, the metal production rates are calculated using the same enrichment models as GASOLINE. However, to find the metal production history, we only consider the metals produced within the main progenitor. To do this, the mass of metals produced between two snapshots was calculated for those star particles within the main progenitor during the latter snapshot. Additional metals are gained through externally accreted gas and stars, frequently as part of a merger. Rates of externally accreted metals were tallied using all gas and star particles that had previously been external to the main progenitor. Gas particles and stars were considered accreted at the time they first entered the galactic disk; however, we determined the metal mass accreted using the metallicity of the gas particles at the time they enter the halo. The metallicity at this earlier time was chosen to ensure that any additional metals picked up as the particle traveled through the halo were not included as external accretion. For all halos, the metals produced by a galaxy overwhelm the metals gained through external accretion, a point that will be further quantified later in this section.

Except in the highest mass galaxies, the mass of metals contained in the ISM and stars is only a small fraction of the total metals produced and accreted from external sources, as also seen in Section 3.2. Therefore, it is clear that metal removal via outflows must be instrumental in setting the metal content of the galaxies. The cumulative mass of metals removed from the disk is shown as the negatively valued gold line. The subset of metals removed that achieve sufficient energy to exceed the escape velocity of the disk, what is generally considered part of an outflow and what we term as "ejected," are shown as the negatively valued red line. An even smaller subset of metals is fully "expelled" from the halo, i.e., they reach a distance farther than the virial radius. The cumulative history of these metals is shown as the blue line. The fraction of metals removed from the disk that satisfy either the ejected or expelled criteria depends strongly on halo mass. The more massive the halo, the higher the energy thresholds for ejection and expulsion, and the less likely a particle achieving a temperature or density sufficient to not be considered removed from the ISM will actually be part of an outflow. An additional distinction between the material removed from the ISM and the subset that is ejected or expelled can be seen in the different shapes of the curves. The mass of metals expelled and ejected track the mass of metals produced since both follow the star formation history (and, therefore, the history of stellar feedback) in the galaxy. In contrast, the mass of metals lost from the disk continues to rise steeply over time as metals continuously rapidly pass in and out of the disk.

Substantial amounts of metal mass are returned to the disk, indicating the importance of gas recycling. We show the cumulative history of all metals reaccreted after leaving the 


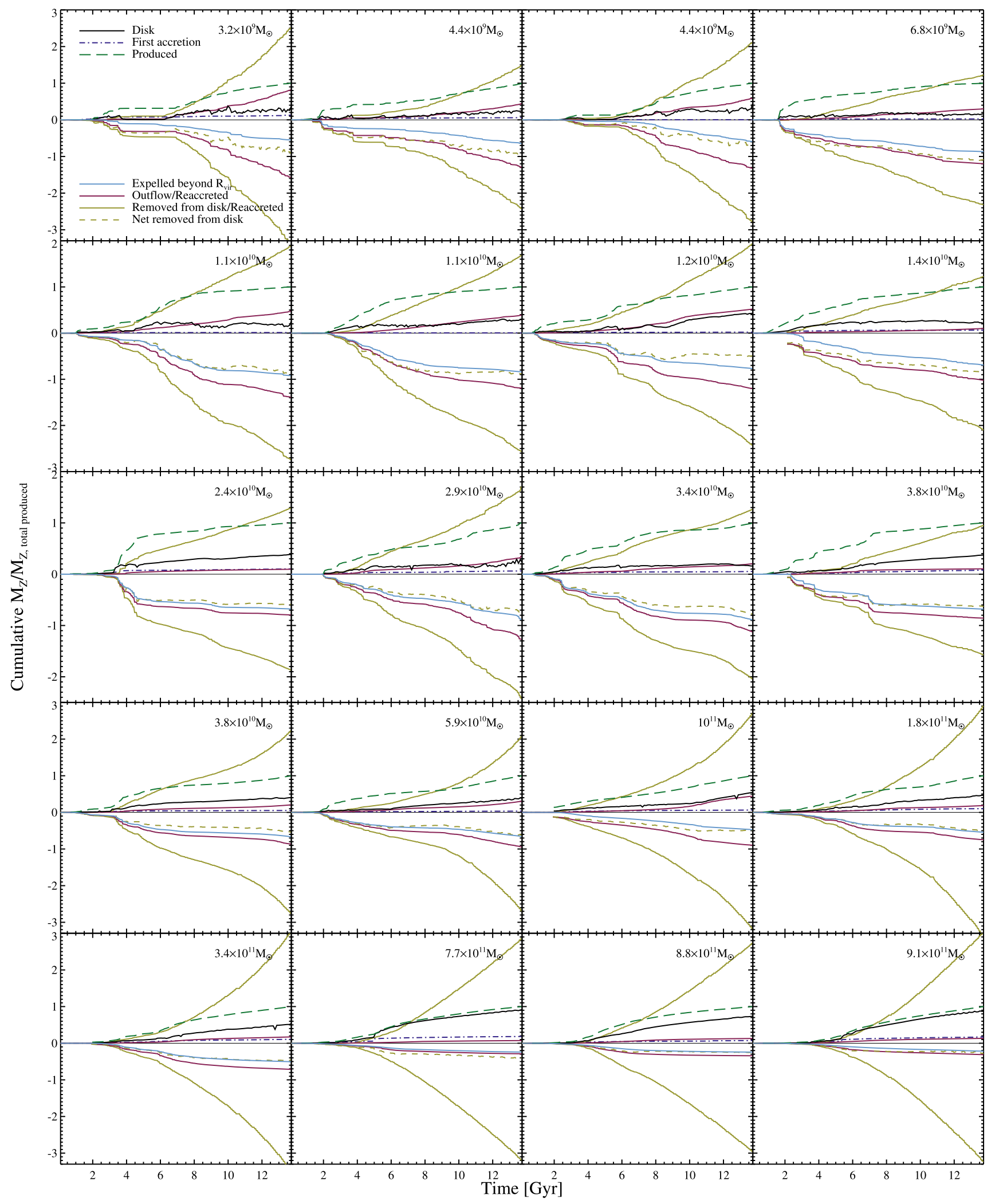

Figure 6. History of the metal buildup within galaxies. All values are scaled by the total mass of metals produced by the stars in the main progenitor by $z=0$. The $z=0$ virial mass of each galaxy is listed in the upper-left corner of each panel. The black solid line represents the metal mass contained within the ISM and stars. All other positive-valued lines show the cumulative histograms of the mass of metals produced by stars (green long-dashed line), accreted externally (purple dotteddashed), reaccreted to the disk after being removed from it (gold), and reaccreted to the disk after being ejected (maroon). The solid negatively valued lines show the cumulative histograms of the mass of metals removed from the disk (gold), ejected from the disk (maroon), and expelled beyond the virial radius (light blue). Additionally, the net metal mass removed from the disk is shown by the dashed gold line. The net metal mass removed was calculated by subtracting the reaccreted metal mass from the removed metal mass. 


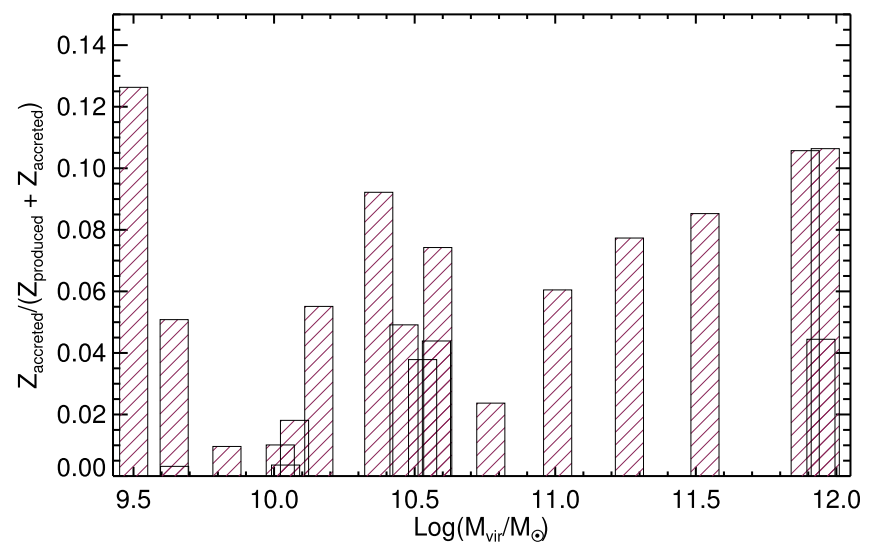

Figure 7. Fraction of metals within $R_{\mathrm{vir}}$ at $z=0$ accreted externally as gas. The denominator includes the sum of all metals produced and all metals accreted as gas from external sources, including through galaxy mergers. In general, a larger fraction of the metals available to higher mass galaxies are accreted externally but the fraction of metals externally accreted is low across the entire mass range.

disk (calculated by subtracting the externally accreted metal mass from the total accretion) as the positively valued gold line. For illustrative purposes, we also show the cumulative history of metals reaccreted as part of particles previously ejected from the disk (red positively valued line). However, this quantity carries the caveat that additional metals may be reaccreted after being ejected by diffusing to other accreted particles. Therefore, this line is a lower limit on the mass of metals actually reaccreted following an outflow. Finally, we show the net mass of metals removed from the disk (cumulative history of metals removed minus the cumulative history of metals reaccreted after removal) as the dashed gold line.

Reaccretion is common across our entire sample. In fact, for all galaxies, there is as much metal reaccretion after removal as there is metal production; for the most massive galaxies, multiple cyclings of gas lead the metal reaccretion mass to exceed the metal production by factors of a few. More massive galaxies tend to have slightly higher rates of reaccretion of removed metals, as shown by the difference between the total mass of metals removed and the net mass of metals removed. Despite the prevalence of recycling, however, much of the metal mass produced remains within the CGM. In fact, the net metal loss exceeds the total metals contained in the disk at all redshifts for any but the three most massive galaxies. While some of these "permanently removed" metals escape the halo, they may also remain within $R_{\mathrm{vir}}$, as seen when the net metal loss exceeds the mass of metals expelled (in other cases where mergers result in the reaccretion of gas from outside the virial radius, the net metal loss from the disk may be less than the total mass of metals expelled).

In the remainder of this section, we further quantify how the amount of material accreted, outflowing, and reaccreted scales with the halo mass. To begin, we quantify the role of external accretion in contributing metals. The fraction of metals that were originally accreted from external sources as gas is shown as a function of halo mass in Figure 7. In this analysis, the mass of metals accreted as gas is compared to the total mass of metals accreted as gas and produced within the main progenitor by $z=0$. As also seen in Figure 6, the fraction of metals externally accreted is uniformly small. However, there appears to be a mass trend, with the most massive galaxies generally

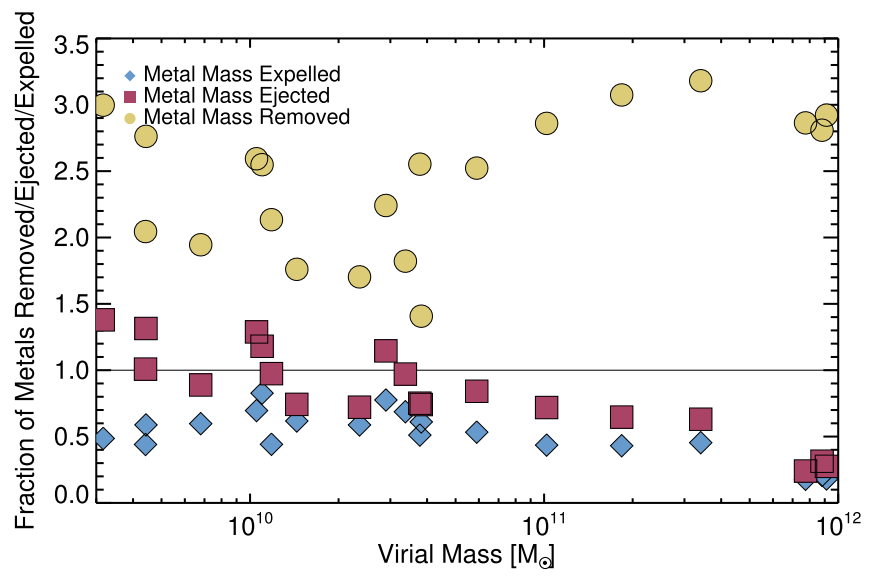

Figure 8. Fraction of metals produced or accreted as gas that were removed from the disk (yellow circles), were ejected from the disk (i.e., became dynamically unbound from the disk; maroon squares), or were expelled beyond the virial radius (blue diamonds). Because metals can be lost again after being reaccreted, this fraction can be greater than one.

accreting a larger fraction of their metals from external sources, probably primarily through mergers.

Figure 8 shows the mass of metals in outflows divided by the total mass of metals available (metals either produced by stars or externally accreted as gas). Because the same metals can exit the disk multiple times, this number is frequently greater than one. In particular, the large amount of metals removed from the disk compared to the amount available highlights the prevalence of both gas removal and gas reaccretion. The likelihood of metals being removed from the disk is largely independent of halo mass, because the possibility of gas being heated is basically independent of the galaxy dynamics. The likelihood of metals being ejected (i.e., part of an outflow), though, is sensitive to mass, because the particles must exceed an energy threshold. As a result, in more massive galaxies, a smaller fraction of either the metals available or the metals removed from the disk is actually considered ejected. Even in the most massive halos, though, $\sim 20 \%$ of the available metals are ejected from the main progenitor. When considering the fraction of metals expelled beyond the virial radius for galaxies with virial masses greater than $\sim 2.5 \times 10^{10} M_{\odot}$, we see a similar, although slightly reduced, trend as for the mass of metals removed from the disk. Specifically, the fraction expelled is $\propto \log \left(M_{\text {vir }} / M_{\odot}\right)^{-0.31}$ while the fraction ejected is $\propto \log \left(M_{\text {vir }} M_{\odot}\right)^{-0.44}$. As seen in Figure 6, in these more massive galaxies, a majority of the gas particles that exit the disk also exit the virial radius, explaining the similarity in the trends. For galaxies with $M_{\text {vir }} \lesssim 3 \times 10^{10} M_{\odot}$, however, the fraction of metals that are expelled beyond the virial radius is roughly constant, even as the fraction that exits the disk increases with decreasing halo mass. As a result, in the lowest mass galaxies, a minority of the metals ejected from the disk are able to escape the virial radius. This effect is especially strong for the three lowest mass galaxies, which have been previously shown to retain metals relatively close to their centers (Figures 3 and 4).

Figure 9 quantifies the fraction of gas reaccreted. Throughout this section, we divide among gas removed from the disk, ejected from the disk, and expelled beyond the virial radius. The fractions of the gas that are reaccreted by $z=2$ and $z=0$ are shown as a function of halo mass. All reaccretion rates rose from $z=2$ to $z=0$ as the greater time elapsed 


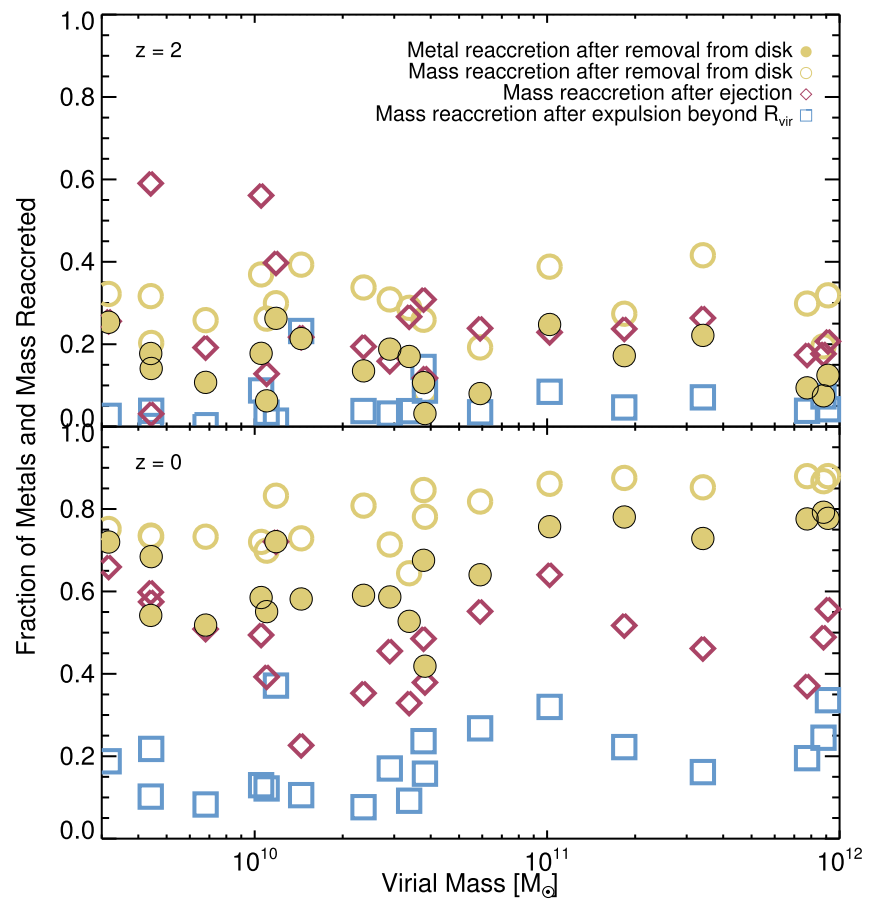

Figure 9. Fraction of metals and mass that are reaccreted by $z=2$ (top panel) and $z=0$ (bottom panel). Open symbols show the mass fraction of particles reaccreted after either being removed from the disk (yellow circles), being ejected from the disk (maroon squares), or expelled beyond the virial radius (blue diamonds). Filled yellow circles show the fraction of metal mass removed from the disk that is later reaccreted.

allowed for more material to cycle back. As anticipated, reaccretion rates are lower for outflowing material satisfying a more stringent energy cut. However, rates of reaccretion at $z=0$ are substantial throughout. In a couple of galaxies, the fraction of mass reaccreted after having left the virial radius even exceeds $30 \%$. At $z=0$, we see slight positive mass trends in the fraction of mass reaccreted after removal from the disk. The fractions of mass reaccreted after ejection or expulsion do not show consistent trends with mass, likely because the mass of the halo is already incorporated into the determination of whether a particle is ejected or expelled.

We also show the metal fraction reaccreted after having been removed from the disk. As in Figure 6, we determine the mass of metals reaccreted by subtracting the mass of metals externally accreted from the total mass of metals accreted onto the disk. ${ }^{8}$ The fraction of metals returned, while high, is noticeably lower than the mass fraction returned. This difference is likely the result of the correspondence between the amount of metals and the amount of energy transferred to gas from SNe. Those particles least likely to return to the disk are those that received the most SN energy and, presumably, relatively large amounts of metals.

\subsection{Outflow Metallicities}

In analytic models of halo enrichment, outflows are frequently assumed to share the same metallicity as the ISM (e.g., Davé et al. 2012; Lilly et al. 2013). However, the correspondence between SN enrichment and the generation of

\footnotetext{
8 We do not show the metal fraction returned by previously ejected or expelled particles because metal diffusion allows metals to be transferred from ejected particles to other particles in the halo.
}

outflows implies that outflows may be metal-enriched compared to the rest of the ISM. Recent observations of outflows by Chisholm et al. (2016) appear to confirm the relative enrichment of outflows. If true, the degree of enrichment would be an important parameter in modeling the metallicities of galaxies. Here, we examine the history of outflow and inflow metallicity in comparison to the ISM.

Figure 10 shows the average metallicities of outflowing and accreting material over time for four representative galaxies spanning our range of masses. Metallicities of both gas removed from the disk and ejected are shown. Ejected material is more metal-rich than material simply removed from the disk because the "ejection" criterion selects for gas particles that receive sufficient $\mathrm{SN}$ feedback to dynamically escape the disk and are, consequently, more likely to also receive large amounts of metals. Both types of outflowing gas, though, are enhanced compared to the ISM, either because of enrichment by the $\mathrm{SNe}$ driving the outflow or because the particles originate in already metal-enhanced areas of ongoing star formation.

We also show the metallicities of accreted material. The metallicity of all accreted gas incorporates both the metals accreted from external galaxies and those reaccreted to the disk. We calculated the metallicity of the subset of that material that is reaccreted by excluding the gas mass that was being accreted to the disk for the first time and those metals that had been accreted onto the halo. As expected, the reaccreted material is an especially metal-enriched subset of the total accretion. Both accretion and reaccretion tend to track the metallicities of the disk material, since that is the primary source of metals in the halo. Occasionally, the metallicity of the reaccreting material appears to be offset in time from the metallicity of the ejected and removed material, e.g., the top-left panel between 2 and 6 Gyr. This temporal offset is a clear signature of fountaining. However, the short reaccretion timescales of $\sim 1$ Gyr (Christensen et al. 2016) and noise in the data make them difficult to identify.

In order to further study mass trends in the relative metal enhancement of outflowing material, we examine the distribution of ejected particle metallicities for all galaxies in Figure 11. This figure shows the histograms of the relative metal enrichment of the ejected material. To determine this enrichment, the metallicity of all ejected gas particles at the snapshot after they leave the disk was divided by the mean metallicity of the ISM at that snapshot. The histograms tend to peak close to one, indicating that ejected gas is most likely to share a similar metallicity to the ISM. However, the histograms also show long tails toward higher levels of metal enrichment, which raises the overall average metal enrichment of outflows. Highly enriched ejected gas particles are most likely the result of the simultaneous transfer of large amounts of metals and energy from nearby $\mathrm{SNe}$, while gas particles with metallicities closer to that of the ambient ISM are more likely to have been ejected through entrainment. The mass trend seen in these histograms may also be explained by differing amounts of entrainment. Histograms for low-mass galaxies peak closer to one than high-mass galaxies, probably because larger amounts of ambient ISM are carried out from these galaxies during outflow events (Christensen et al. 2016), although a more homogeneous distribution of metals in the ISM of dwarf galaxies could have a similar effect. 

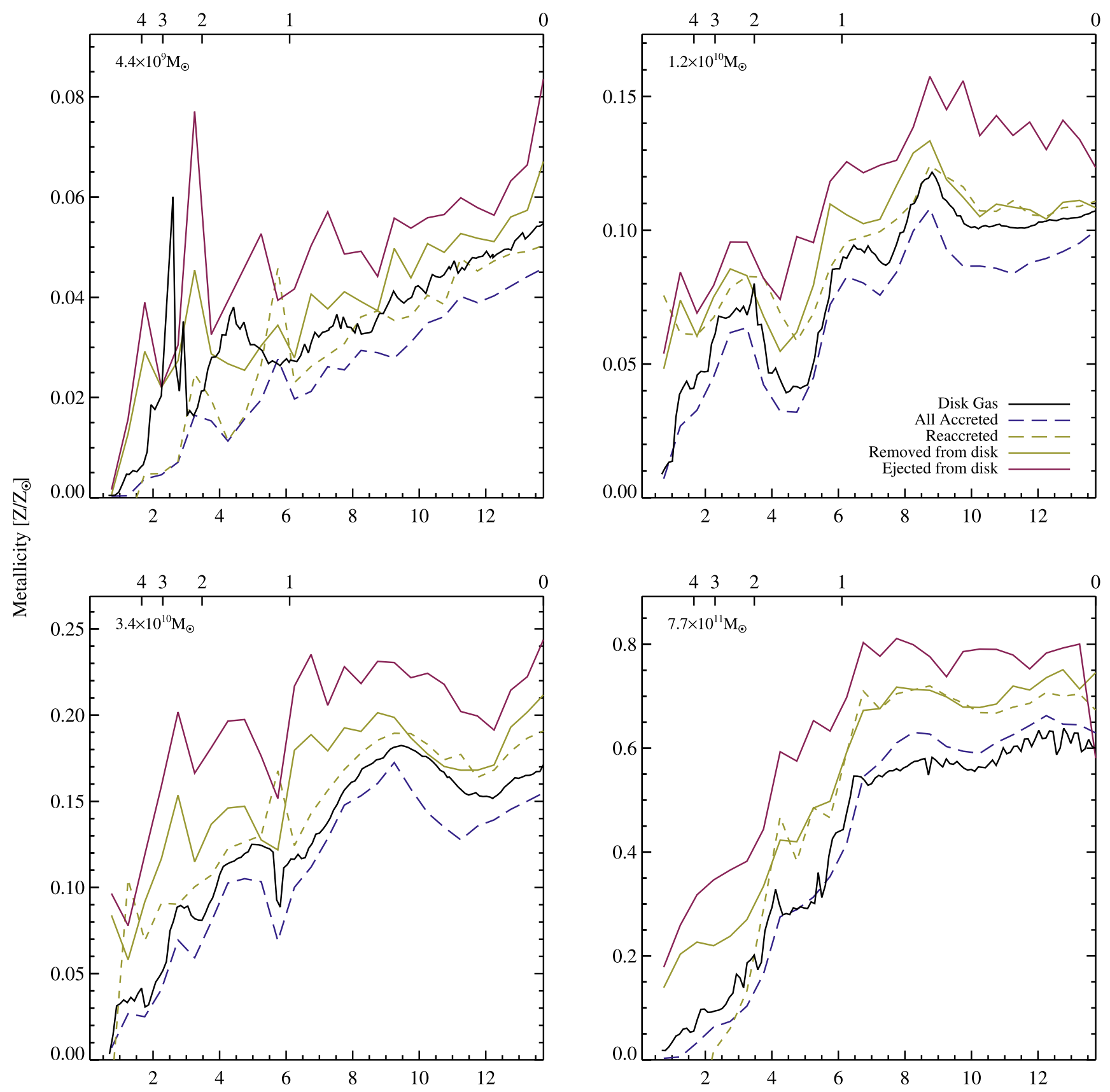

Time [Gyr]

Figure 10. History of average metallicities of outflowing and accreting material for four example galaxies spanning a range of masses. The virial masses of each galaxy are shown in the top-left corners of the panels. The solid black line shows the average metallicity of the ISM. Solid colored lines show the average metallicity of gas that is removed from the disk (gold) and ejected such that it dynamically escapes the disk (maroon). The long-dashed purple line follows the average metallicity of all accreted and reaccreted material. The short-dashed gold line shows the average metallicity of material reaccreted onto the disk.

We further quantify the mean metallicities of outflows at different redshifts (Figure 12, top panel). Unsurprisingly, the ejecta metallicity, like that of the disk gas, increases with halo mass. There is little to no redshift evolution in the relationship between ejecta metallicity and virial mass despite the evolving mass-metallicity relation.

Dividing the mean metallicity of the ejected gas by that of the ISM at the time when it was ejected provides a measurement of the relative enrichment of the ejecta at different redshifts (Figure 12, bottom panel). We see a large range of enrichment levels and a few cases where the outflow metallicity is actually lower than the ambient ISM. We also do not observe a clear metal enrichment trend with mass, although there is some evidence that intermediate-mass galaxies have the highest level of metal enrichment. Conversely, the very lowest mass galaxies had some of the smallest amounts of relative metal enrichment, possibly arising from higher rates of entrainment in these galaxies. Therefore, the low metallicities of dwarf galaxies are not because they preferentially ejected metals compared to more massive galaxies but are rather because they are more efficient at ejecting material in general, as will be explored in the next section. Metal enrichment levels 


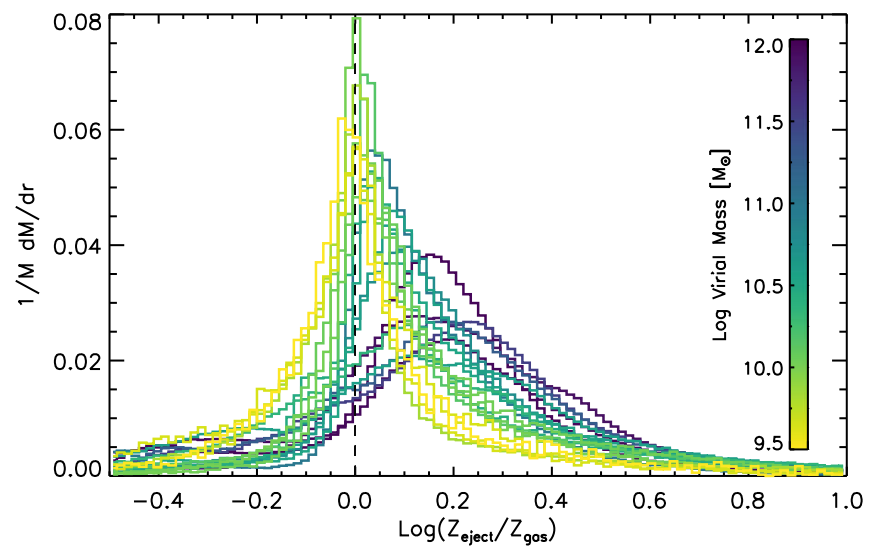

Figure 11. Normalized histogram of the metallicities of ejected gas particles divided by the mean metallicity of the ISM at the time of ejection. Each curve represents all gas ejected over the history of a single galaxy, with different colors corresponding to different redshift zero virial masses. Most curves peak close to one with a long tail toward higher metal enrichment. The curves for more massive galaxies tend to peak at higher levels of metal enrichment, perhaps indicating reduced amounts of entrained material in outflows from those galaxies.

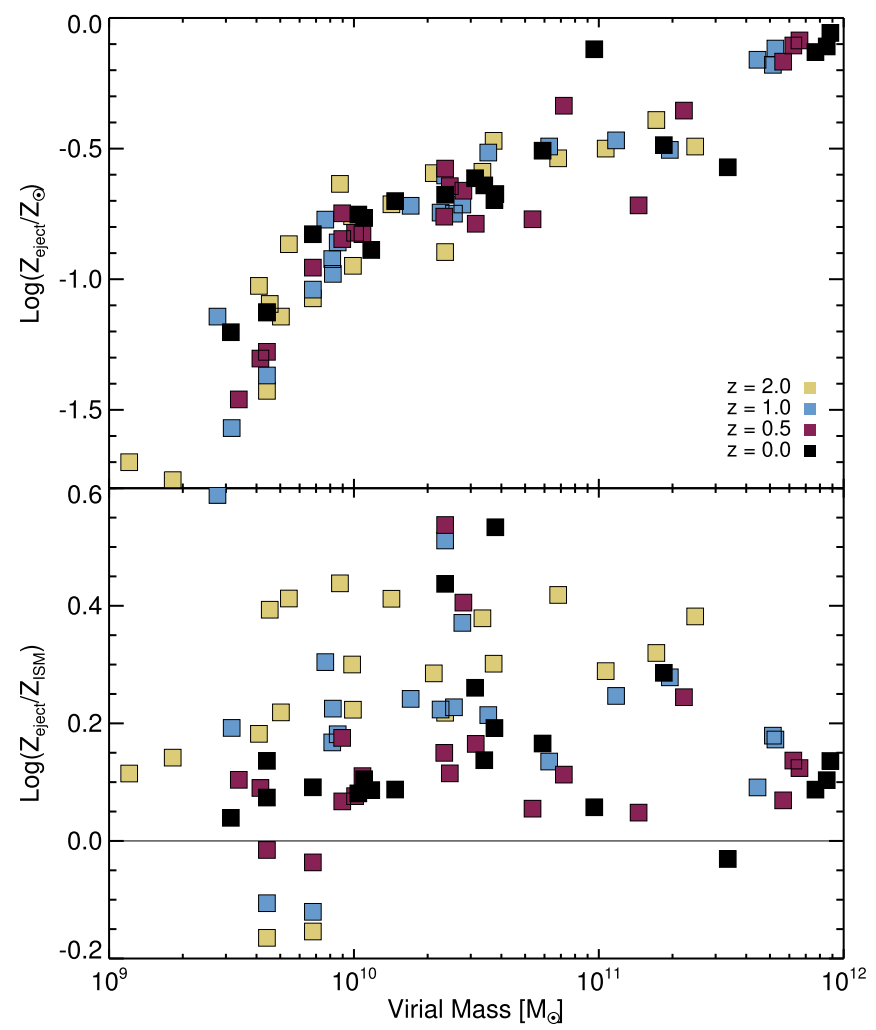

Figure 12. Metallicity of ejected material. The top panel shows the mean metallicity of the gas ejected at different redshifts as a function of virial mass. There is a trend toward increasing metallicity with increasing virial mass, as is expected from the MZR. There is no observed redshift evolution in the relationship between outflow metallicity and galaxy mass. The bottom panel shows the mean metallicity of the ejected material normalized by the mean metallicity of the disk gas at that redshift. Compared to the ambient gas, outflows generally involve much more highly enriched material. The relative enrichment of the ejected material increases with $z$.

did tend to be higher at $z=2$ and, to a lesser extent, at $z=1$. At these redshifts, the ISM metallicity would have been lower, leading to a greater difference between it and the recently enriched gas near SNe.

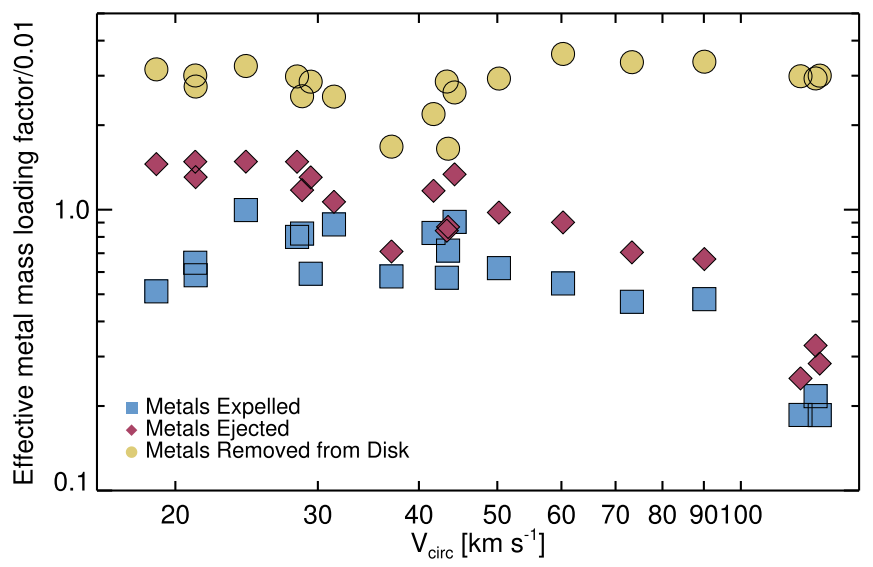

Figure 13. Total mass of metals lost over the history of the galaxy normalized by the total stellar mass formed, also known as the effective metal mass loading, shown as a function of galaxy circular velocity. The effective metal mass loading shown here is scaled by the stellar yield for the Kroupa et al. (1993) IMF: 0.01. Different symbols represent different selection criteria for outflowing material: gold circles include all material that leaves the disk, maroon squares show only ejected material that exceeds the escape velocity of the disk, and blue diamonds show only material that is eventually expelled beyond the virial radii. Tightening the criteria for outflow identification to require gas to either dynamically escape the disk or leave the halo both reduces the effective metal mass loading and introduces a mass dependency. While similar amounts of metals are removed from the ISM because of stellar feedback, the deeper potential wells of higher mass galaxies result in a smaller mass of metals in outflows per stellar mass formed.

By $z=0$, the average relative enrichment was only a factor of 1.5. These redshift zero metal enrichment results for the most massive galaxies are consistent with observations of NGC $6090\left(M_{*}=10^{10.7} M_{\odot}\right)$, which showed a factor of $1.3=10^{0.11}$ greater metallicity in outflows than the ISM (Chisholm et al. 2016). Similar, although slightly smaller, amounts of metal enrichment were also found by Muratov et al. (2017) in their simulations. Specifically, for redshifts $4<z<0$, they found that winds were generally more metal-enriched by a factor of $\sim 1-1.5$. As in this work, they also found a trend toward greater metal enrichment at higher redshift. Likewise, they did not observe a general mass dependency but did observe that outflows with no metal enrichment (or even metal depletion) compared to the interstellar media came from the smallest galaxies.

\subsection{Metal Mass Loading}

The efficiency of galaxies at expelling their metals can be quantified as the "metal mass loading" factor, i.e., the rate at which metals are ejected divided by the rate at which star formation occurs. An effective metal mass loading representing the metal loss integrated over the history of the galaxy can be found by dividing the total mass of metals in outflows by the total mass of stars formed. Figure 13 shows the effective metal mass loading as a function of circular velocity for several different ways of identifying outflows: all gas removed from the disk, gas that exceeds the escape velocity for the disk ("ejected"), and gas that is expelled beyond the virial radius. The metallicities used in these calculations are the metallicities of gas particles at the snapshot immediately prior to their removal. As would be expected, increasingly stringent criteria for identifying outflowing material result in lower effective metal mass loading rates. Requiring that the outflowing material satisfy an energy criterion, either exceeding the escape 


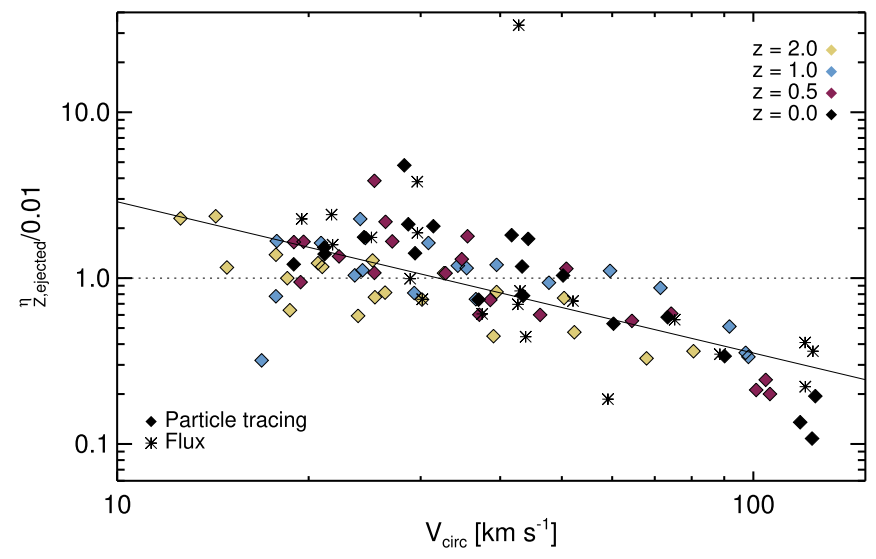

Figure 14. Mass of metals ejected in particle-tracing-selected outflows divided by the mass of stars formed in 1 Gyr time bins (i.e., the instantaneous metal mass loading) as a function of galaxy circular velocity (filled symbols). Measurements were made at $z=0,0.5,1$, and 2. A logarithmic fit to the ejection data at all redshifts is shown by the solid line. The asterisks show the instantaneous metal mass loading at $z=0$ as calculated by measuring the flux through a spherical shell of radius $0.25 R_{\text {vir }}$ for comparison. The instantaneous metal mass loading factors shown here are scaled by a typical stellar yield for a Kroupa et al. (1993) IMF: 0.01.

velocity or leaving the virial radii, also introduces a mass dependency to the effective metal mass loading. While similar amounts of metals per stellar mass formed are removed from the disk across the entire mass range of galaxies, the deeper potential wells of more massive galaxies result in smaller fractions of the metals able to dynamically escape the disk. The result is substantially lower efficiencies of metal loss through outflows in more massive galaxies.

Figure 14 shows a more instantaneous metal mass loading factor, $\eta_{\text {metals }}$, as determined using particle-tracing-selected outflows (filled symbols) and through a calculation of the metal flux (asterisks). In the case of the particle-tracing-selected outflows, metal loss and star formation rates are calculated for 1 Gyr time bins centered on each of four redshifts $(z=0,0.5$, 1.0, and 2.0). We only consider the metals carried by gas exceeding the disk escape velocity ("ejected"), as this criterion best selects for gas generally considered part of outflows. A power-law fit to the data for all redshifts results in $\eta_{\text {metals }} \propto v_{\text {circ }}^{-0.91}$. A similar fit to the total mass loading function in Christensen et al. (2016) had a $v_{\text {circ }}^{-2.2}$ dependency. The shallower dependency of the metal mass loading, also detected in Creasey et al. (2015), is the result of the MZR. The relation can be explained by scaling the mass loading relation by the metallicity of the outflow. As seen in Figure 12, outflow metallicity increases with virial mass following a scaling of approximately $Z_{\text {ejecta }} \propto M_{\text {vir }}^{0.46} \propto v_{\text {circ }}^{1.4}$. This scaling is very similar to that observed for the low end of the MZR, as would be anticipated from the lack of mass scaling in the relative metal enhancement of the ejecta. Therefore, $\eta_{\text {metals }}=Z_{\text {ejecta }} \eta_{\text {total }} \propto v_{\text {circ }}^{1.4} v_{\text {circ }}^{-2.2} \sim v_{\text {circ }}^{-0.91}$. As a result, while dwarf galaxies have low metallicities and they do not preferentially eject metals compared to higher mass galaxies, their tendency to eject more mass overall results in high metal mass loading factors.

Metal mass loading factors were previously measured in Muratov et al. (2017) by calculating the metal flux through a $0.25 R_{\text {vir }}$ sphere surrounding the center of the galaxy. In order to draw a direct comparison to their work, we also show the metal mass loading factor as determined from the flux, $\eta_{\text {flux }}$, following the method in Muratov et al. (2017). Specifically, we identified all particles with outward radial velocities within a spherical shell of inner radius $0.2 R_{\mathrm{vir}}$ and outer radius $0.3 R_{\mathrm{vir}}$ as part of an outflow. Using these particles, $\eta_{Z \text {,flux }}$ is defined to be

$$
\eta_{Z, \text { flux }}=\frac{1}{\dot{M}_{\mathrm{SFR}}} \frac{\partial M}{\partial t}=\frac{1}{\dot{M}_{\mathrm{SFR}}} \Sigma v_{\mathrm{rad}} Z_{\mathrm{SPH}} m_{\mathrm{SPH}} / d L
$$

where $M_{\mathrm{SFR}}$ is the star formation rate averaged over $100 \mathrm{Myr}$, $v_{\text {rad }}$ are the radial velocities of the outflowing gas particles, $Z_{\mathrm{SPH}}$ are the metallicity of the particles, $m_{\mathrm{SPH}}$ are their masses, and $d L$ is the width of the spherical shell $\left(0.1 R_{\mathrm{vir}}\right)$. Similarly to Muratov et al. (2017), we calculated $\eta_{Z \text {,flux }}$ at five different snapshots in the last $3 \mathrm{Gyr}$ to reduce the noise. We found that calculating the metal mass loading factor using flux through a sphere, rather than particle tracing, introduces a greater amount of scatter. However, the trend and scaling are similar for both methods, indicating that our metal mass loading factors are largely insensitive to the method used to measure them.

Muratov et al. (2017) found a metal mass loading factor approximately equal to the $\mathrm{SNe}$ II yield (0.02) for their simulations across all masses and redshifts, which they interpret as implying that all metals produced through $\mathrm{SNe}$ II are immediately ejected, at least temporarily. In contrast, we find a definite mass dependency. We calculate metal mass loading factors for dwarf galaxies slightly higher than simulation yields and metal mass loading factors for MilkyWay-mass galaxies almost a factor of 5 lower than the yields. The fact that the metal mass loading factors are slightly higher than the yields in our dwarf galaxies likely indicates that entrainment is removing (at least temporarily) additional metals beyond those produced by the $\mathrm{SNe}$, while the lower metal mass loading factors for more massive galaxies demonstrate that $\mathrm{SNe}$ are incapable of removing most of the produced metals from the more massive disks. Both of these phenomena are likely absent in the Muratov et al. (2017) FIRE simulations.

\section{Discussion}

\subsection{Generation of the Mass-Metallicity Relation}

The MZR can be explained through a combination of inflow, outflow, and recycling rates, in addition to star formation efficiencies. Here we discuss the role of these processes in establishing the MZR in the simulated galaxies presented here. As a framework, we modify the analytic model outlined in Appendix C of Peeples \& Shankar (2011) to include reaccretion (see Zahid et al. 2014 for a similar modification).

One can write the instantaneous change in the metal mass, $\dot{M}_{Z}$, as

$$
\begin{aligned}
\dot{M}_{Z}= & Z_{\mathrm{IGM}} \dot{M}_{\mathrm{acc}}-Z_{\mathrm{ISM}} \dot{M}_{\mathrm{SFR}}+Z_{\mathrm{ej}} \dot{M}_{\text {recy }} \\
& -Z_{W} \dot{M}_{W}+Z_{\text {reaccr }} \dot{M}_{\text {reaccr }},
\end{aligned}
$$

where $Z_{\mathrm{IGM}}$ is the metallicity of the IGM, $\dot{M}_{\mathrm{acc}}$ is the rate of external mass accretion, $Z_{\text {ISM }}$ is the metallicity of the ISM, $\dot{M}_{\mathrm{SFR}}$ is the star formation rate, $Z_{\mathrm{ej}}$ is the metallicity of gas being returned to the ISM by stars, $\dot{M}_{\text {recy }}$ is the rate at which mass is returned from ISM by stars, $Z_{W}$ is the metallicity of the galactic wind, $\dot{M}_{W}$ is the rate of mass outflow, $Z_{\text {reaccr }}$ is the metallicity of the reaccreted material, and $\dot{M}_{\text {reaccr }}$ is the rate of 
mass reaccretion. We can define a nucleosynthetic yield:

$$
y=Z_{\text {ej }} \frac{\dot{M}_{\text {recy }}}{\dot{M}_{\text {SFR }}}=Z_{\text {ej }} f_{\text {recy }} .
$$

For the IMF and theoretical yield models used in this paper, $y \approx 0.01$. By also defining a metal accretion efficiency, $\zeta_{a}=\frac{Z_{\mathrm{IGM}}}{Z_{\mathrm{ISM}}} \times \frac{\dot{M}_{\text {acc }}}{\dot{M}_{\mathrm{SFR}}}$, Equation (3) can be simplified to

$$
\begin{aligned}
\dot{M}_{Z}= & \dot{M}_{\mathrm{SFR}} \\
& \left(y+Z_{\mathrm{ISM}}\left(\zeta_{a}-1-\frac{Z_{W} \dot{M}_{W}-Z_{\text {reaccr }} \dot{M}_{\text {reaccr }}}{Z_{\mathrm{ISM}} \dot{M}_{\mathrm{SFR}}}\right)\right) .
\end{aligned}
$$

Similarly to $\zeta_{a}$, we define a net metal expulsion efficiency:

$$
\zeta_{\text {net }}=\frac{Z_{W} \dot{M}_{W}-Z_{\text {reaccr }} \dot{M}_{\text {reaccr }}}{Z_{\mathrm{ISM}} \dot{M}_{\mathrm{SFR}}}
$$

Provided reaccretion happens on reasonably short time periods, $\dot{M}_{\text {reaccr }}=f_{\text {reaccr }} \dot{M}_{W}$, and

$$
\zeta_{\text {net }}=\frac{\dot{M}_{W}\left(Z_{W}-Z_{\text {reaccr }} f_{\text {reaccr }}\right)}{Z_{\text {ISM }} \dot{M}_{\text {SFR }}},
$$

where $f_{\text {reaccr }}$ is the fraction of material leaving the disk that is later reaccreted. If there were to be no reaccretion of wind material, $f_{\text {reaccr }}$ would be equal to 0 and $\zeta_{\text {net }}$ would reduce to $\zeta_{\text {net }}=\frac{Z_{W}}{Z_{\mathrm{ISM}}} \times \frac{\dot{M}_{W}}{\dot{M}_{\mathrm{SFR}}}$. By substituting $\zeta_{\text {net }}$ into Equation (5), it reduces to

$$
\dot{M}_{Z}=\dot{M}_{\mathrm{SFR}}\left(y+Z_{\mathrm{ISM}}\left(\zeta_{a}-1-\zeta_{\text {net }}\right)\right) \text {. }
$$

The scaling of $\zeta_{\text {net }}$ with halo mass uniquely determines the MZR if a stellar mass-halo mass relation is adopted and if $y$ and $\zeta_{a}$ are assumed to be independent of halo mass. To simplify further, one could assume that $\zeta_{a} \approx 0$, an assumption supported by the minimal metallicity of externally accreted material measured in our simulations (Figure 6). Similarly, $y$ is independent of time for a given IMF.

By combining equations governing the instantaneous change in total mass and metal mass and assuming a power-law relationship between gas fraction and stellar mass such that $F_{g}=\frac{M_{g}}{M_{*}}=K_{f} M_{*}^{-\gamma}$, one can write

$$
\frac{d Z_{g}}{d Z_{*}}=\frac{y+Z_{g}\left(\zeta_{a}-1-\zeta_{\text {net }}-F_{g}(1-\gamma)\right)}{M_{g}\left(1-f_{\text {recy }}\right)}
$$

following the derivation in Appendix C of Peeples \& Shankar (2011). For a given relationship between $\zeta_{\text {net }}$ and $M_{*}$, this equation can be integrated with respect to $M_{*}$ to produce an MZR.

In Figure 15, we show $\zeta_{\text {net }}$ versus $v_{\text {circ }}$ for our simulations. To calculate $\zeta_{\text {net }}$, we determined the net mass removed from the disk across $1 \mathrm{Gyr}$ time bins at four different redshifts and scaled the values by the ISM metallicity and star formation rate at those times. Excluding the 3 out of 40 instances when the metal influx exceeded the metal outflux, we find that the data are well fit by a power law: $\zeta_{\text {net }}=3.4 v_{\text {circ }}^{-1.7}$. The scaling with $v_{\text {circ }}$ is less extreme than the mass loading factor, $\eta_{\text {total }} \propto v_{\text {circ }}^{-2.2}$, but more so than the metal mass loading factor, $\eta_{\text {metals }} \propto v_{\text {circ }}^{-0.91}$. As $f_{\text {reaccr }}$, the fraction of material reaccreted after leaving the disk, is relatively constant with halo mass (Figure 9), the more extreme mass scaling for $\zeta_{\text {net }}$ than $\eta_{\text {metals }}$ must arise from the additional factor of $1 / Z_{\text {ISM }}$ in $\zeta_{\text {net }}$. Since $Z_{\text {ISM }}$ is smaller in low-mass

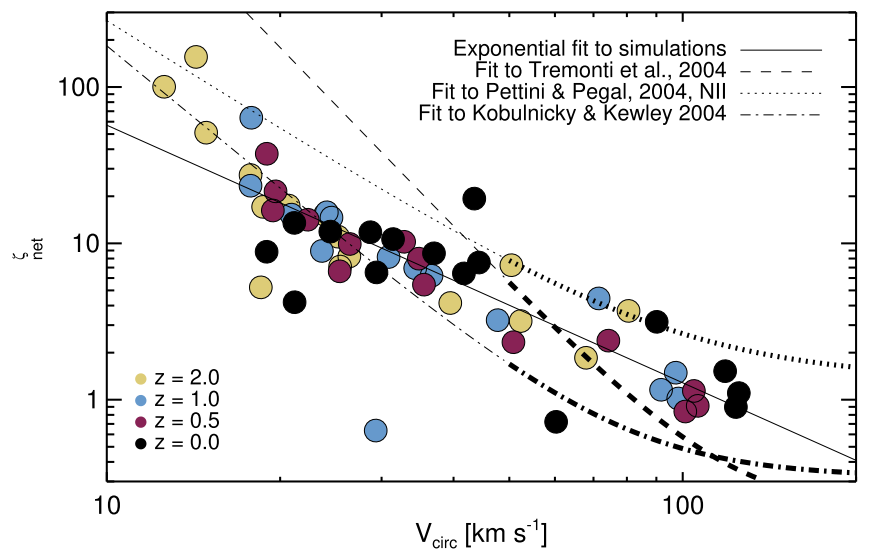

Figure 15. Net metal expulsion efficiency, $\zeta_{\text {net }}$, calculated by dividing the net mass loss from the disk by the total stellar mass formed and scaling by the ISM metallicity and star formation rate, vs. the circular velocity of the galaxy. Measurements are shown for $1 \mathrm{Gyr}$ bins at four different redshifts. The solid black line shows a power-law fit to the data. Thick patterned black lines show the predicted relationship between $\zeta_{W}$ and $v_{\text {circ }}$ from Peeples \& Shankar (2011), assuming observed MZRs based on three different indicators, Tremonti et al. (2004; not adjusted, dashed), Pettini \& Pagel (2004; dotted), and Kobulnicky \& Kewley (2004; dotted-dashed). The thin patterned black lines show the extrapolation of the predicted relationships below the observed range.

galaxies, this factor results in $\zeta_{\text {net }}$ having a stronger mass dependency.

For comparison, we also show three best-fit functions for $\zeta_{\text {net }}$ to different observed MZRs from Peeples \& Shankar (2011). These functions were based on SDSS data of local, starforming galaxies, compiled and adjusted for different metallicity indicators by Kewley \& Ellison (2008). The indicators, Tremonti et al. (2004), Pettini \& Pagel (2004) N II, and Kobulnicky \& Kewley (2004), were chosen to represent the range of possible values. ${ }^{9}$ The fits shown have been extrapolated (shown with a thinner line) beyond the $M_{*}=10^{8.5} M_{\odot}\left(v_{\text {circ }} \sim 50 \mathrm{~km} \mathrm{~s}^{-1}\right)$ limit used in Kewley \& Ellison (2008).

For galaxies with $v_{\text {circ }}>50 \mathrm{~km} \mathrm{~s}^{-1}, \zeta_{\text {net }}$ lies within what would be expected for different metallicity indicators, although notably slightly lower than the Pettini \& Pagel (2004) N II calibration we compared to previously in this paper. For less massive galaxies, $\zeta_{\text {net }}$ is lower than what would be expected by extrapolating fits from more massive galaxies. However, a slightly flatter MZR at these masses is consistent with data from observed dwarf galaxies by Lee et al. (2006), explaining why the simulations are still able to match the observed MZR at lower masses. This analysis illustrates the role of outflows in our simulations in setting the MZR.

\subsection{Comparison with Other Simulations}

The net metal expulsion efficiency, $\zeta_{\text {net }}$, encapsulates a large amount of physics that together define the rate of metal loss from the disk for a given halo mass, including the mass loading factors, rates of recycling, and the relative metal enrichment of outflow and reaccretion. Other simulations with different outflow mechanics may achieve similar fits to the MZR by having similar values of $\zeta_{\text {net }}$. For example, higher rates of metal outflows may be balanced by higher rates of metal recycling. This can happen either because of a greater total amount of

\footnotetext{
9 Note that in comparing our data to the MZR in Section 3.1, we showed data using or adjusted to the Pettini \& Pagel (2004) N II calibration when possible.
} 
recycling or because of a smaller difference between the inflow and outflow metallicity. Alternatively, higher enrichment of outflowing material relative to the ISM could compensate for lower mass loading factors. Additionally, different mass scalings of the mass loading factors, amount of reaccretion, and relative enrichment of outflows may conspire to produce similar scalings for $\zeta_{\text {net }}$. We discuss here the numerics of our simulations in comparison to different feedback models and highlight the measurements that could help break the degeneracy.

The delayed-cooling, blastwave model for SN feedback used in these simulations naturally produces energy-driven wind scalings (Christensen et al. 2016), which is consistent with those models generally assumed for lower halo masses in simulations with hydrodynamically decoupled winds (Ford et al. 2014; Vogelsberger et al. 2014). However, compared to simulations using hydrodynamically decoupled winds, our simulations result in higher amounts of preventative feedback and lower rates of recycling, presumably because of the higher temperature of the halo gas (Christensen et al. 2016). For instance, Oppenheimer \& Davé (2008) found that about $85 \%$ of wind particles were reaccreted with recycling timescales between $10^{10}$ and $10^{8}$ years, depending on halo mass (Oppenheimer et al. 2010), while the blastwave feedback model used here resulted in about half the outflow gas reaccreting at least once with timescales of $10^{9}$ years, independent of halo mass (Christensen et al. 2016). Given these differences, we would anticipate hydrodynamically decoupled wind simulations that also reproduce the MZR to have higher values of $\dot{M}_{W}$ and $\dot{M}_{\text {reaccr }}$ than our simulations.

Our simulations behave more similarly to those presented in Muratov et al. (2017), who used a feedback model based on energy and momentum injection according to stellar population synthesis models. Those simulations had reaccretion times of a few hundred million years for all halo masses (Anglés-Alcázar et al. 2017). While those recycling times are shorter than the $10^{9}$ year timescales measured for our sample of simulations, the similar lack of a mass dependency is striking. As expected from the shorter recycling times, the fraction of outflowing mass reaccreted was also higher: $50 \%-80 \%$ of gas, compared to the $25 \%-65 \%$ of ejected material reaccreted in our simulations. The simulations in Muratov et al. (2017) also have generally higher mass loading factors with a steeper mass dependency. Their degree of wind metal enrichment is lower and has a shallower mass dependency than in our simulations. Those two factors combined result in a flat metal mass loading factor compared to the $\eta_{\text {metals }} \propto v_{\text {circ }}^{-0.91}$ scaling presented here. We would expect the higher reaccretion rates they find to be balanced by their higher metal mass loading factors, resulting in values of $\zeta_{\text {net }}$ similar to ours. However, while some of the difference in reaccretion is almost certainly the result of the different feedback models, these studies do not select outflows the same way, introducing uncertainty in making the comparison.

The range of numerical models able to reproduce the MZR within the observational uncertainties raises the question of what observations could be used to distinguish between them. The rates and metallicities of inflowing and outflowing material are one set of key quantities; however, selecting the same gas in simulations that observers detect is not trivial. The amount, temperature, and density of metals in the CGM can provide a complementary constraint to the MZR. For example, the distance metals are spread to varies depending on feedback models and should be closely related to the timescales and likelihood of reaccretion. However, this comparison is limited both by the difficulty in making and interpreting observations of the CGM and the difficulty in creating a simulation that can correctly reproduce the multiphase CGM. Furthermore, typical simulations do not resolve the low-density CGM to high levels, so it is possible that substantial amounts of the CGM could be in structures below the resolution limit.

\section{Conclusions}

In this paper, we analyze the drivers of the metal distribution in a set of 20 simulated galaxies spanning two and a half orders of magnitude in halo mass that match the observed characteristics, including the stellar and gaseous MZR. We follow the accumulation of metals in galaxies by tracking their metal production and by identifying instances of metal accretion and loss. This analysis enables us to determine the role of galactic winds and reaccretion in determining the mass of metals within different components of a galaxy and its CGM.

1. Gas outflows are highly effective at removing metals from galactic disks. In the lowest mass galaxies, as little as $10 \%$ of the metals produced may remain within the ISM or stars at $z=0$, while that fraction rises to as much as $\sim 50 \%$ for Milky-Way-mass galaxies. This mass trend is dominated by the stars. While the ISM retains a similar range of fractions (between $5 \%$ and $25 \%$ ) of the produced metals across galaxy mass, the fraction locked in stars rises steeply with galaxy mass. Those metals that do exit the disk of the galaxy are widely dispersed, with the majority lying beyond the virial radius. Because of their deeper potential wells, more massive galaxies are generally better able to retain their metals within their virial radius and show lower dispersal of metals. However, this mass trend becomes complicated for dwarf galaxies, as the very lowest mass galaxies are able to retain a moderate fraction of their metals, despite their shallow potentials. In these galaxies, extremely low star formation rates are likely responsible for the reduced metal loss.

2. The history of metal enrichment of the ISM and stars largely follows the history of metal production by stars with less than $10 \%$ of metals at $z=0$ coming from externally accreted gas or stars. Large amounts of metals cycle rapidly in and out of the disk with the cumulative history of gas loss sometimes exceeding the mass of metals produced by a factor of 3 . The majority of these metals (generally between $50 \%$ and $80 \%$ ), however, are quickly returned to the disk. The fraction of these metals that become dynamically unbound from the disk ("ejected") is much higher in low-mass than high-mass galaxies. These ejected metals are more likely to permanently remain outside of the disk.

3. Ejected material tends to be somewhat more metal-rich than the ambient ISM, because gas that receives energy from $\mathrm{SNe}$ most likely also received metals from the same stellar population. This effect is largely independent of galaxy mass and is generally stronger at high redshift when the ISM metallicity at a given virial mass would have been lower. Gas that is removed from the disk, but 
not necessarily considered part of an outflow, also shows some amount of metal enrichment, especially at early times, but the effect is reduced, presumably because this material includes gas not as strongly heated by $\mathrm{SNe}$. Because of dilution by low-metallicity external accretion, the average metallicity of accreted material is lower than outflowing material and, in most cases, the ISM. However, even when excluding external accretion, the metallicity of material reaccreted after being removed from the disk is lower than the metallicity of the removed material. This difference in metallicity indicates a tendency for some highly enriched material to remain outside the disk.

4. The metal mass loading factor, $\eta_{\text {metals }}=Z_{W} \frac{\dot{M}_{W}}{\text { SFR }}$, shows a power-law dependency on virial velocity, similar to the standard mass loading factor. However, we observe a flattening of the power from $a=-2.2$ to $a=-0.91$ when comparing the metal mass loading to the standard mass loading. This flattening can be entirely explained by the reduced metallicity of the ISM in lower mass galaxies. Therefore, while low-mass galaxies have lowmetallicity ISM and are no more likely to preferentially eject metals than higher mass galaxies, their exceptionally high mass loading factors still produce high rates of metal loss per stellar mass formed.

5. The MZR can be explained using an alternative depiction of the metal expulsion efficiency, $\zeta_{\text {net }}=\frac{Z_{W} \dot{M}_{W}-Z_{\text {reaccr }} \dot{M}_{\text {reacer }}}{Z_{\text {ISM }} \dot{M}_{\text {SFR }}}$, which scales the net metal loss rate from winds by the star formation rate and ISM metallicity. We find that $\zeta_{\text {net }}$ scales as $v_{\text {circ }}^{-1.7}$, which is consistent with what would be expected from the observed MZR. We find the outflowing material to be enriched to the same degree relative to the ISM independent of stellar mass, while reaccretion rates of metals removed from the disk are only slightly higher in more massive galaxies. As a result, the metal expulsion efficiency, $\zeta_{\text {net }}$, shares a similar, though slightly shallower, scaling with $v_{\text {circ }}$ as the mass loading factor.

These results illustrate how outflows and gas recycling, in combination with accretion and varying star formation efficiency, can together produce the MZR. The simulations naturally produce the mass loading factors of energy-driven winds, with a slight metal enhancement and moderate rates of reaccretion across all halo masses. However, this is not a unique solution to producing the MZR, and further comparisons to the metal content, distribution, and thermodynamic structure of the CGM are necessary to constrain how exactly gas transfer between the ISM and CGM determines the baryonic and metal content of galaxies.

The authors are grateful to the referee for the thoughtful critique that improved this paper. The authors would like to thank Rachel Somerville for her insightful feedback. Resources supporting this work were provided by the NASA High-End Computing (HEC) Program through the NASA Advanced Supercomputing (NAS) Division at Ames Research Center as well as the Texas Supercomputing Center. T.Q. acknowledges support from NSF grant NSF AST-1514868. R.D. acknowledges support from the South African Research Chairs Initiative and the South African National Research Foundation.
This research was also supported in part by the NSF under grant No. NSF PHY11-25915.

\section{ORCID iDs}

Charlotte R. Christensen (i) https://orcid.org/0000-00016779-3429

Romeel Davé (iD https://orcid.org/0000-0003-2842-9434

Alyson Brooks (iD https://orcid.org/0000-0002-0372-3736

Sijing Shen (iD https://orcid.org/0000-0001-8523-1171

\section{References}

Abel, T., Anninos, P., Zhang, Y., \& Norman, M. L. M. 1997, NewA, 2, 181 Andrews, B. H., \& Martini, P. 2013, ApJ, 765, 140

Anglés-Alcázar, D., Faucher-Giguère, C.-A., Kereš, D., et al. 2017, MNRAS, 470, 4698

Black, J. H. J. 1981, MNRAS, 197, 553

Blanton, M. R., \& Roweis, S. 2007, AJ, 133, 734

Bordoloi, R., Tumlinson, J., Werk, J. K., et al. 2014, ApJ, 796, 136

Bouche, N., Murphy, M. T., Kacprzak, G. G., et al. 2013, Sci, 341, 50

Brook, C. B., Stinson, G., Gibson, B. K., et al. 2012, MNRAS, 419, 771

Brooks, A. M., Governato, F., Booth, C., et al. 2007, ApJL, 655, L17

Brooks, A. M., Papastergis, E., Christensen, C. R., et al. 2017, ApJ, 850, 97

Cen, R. 1992, ApJS, 78, 341

Chabrier, G. 2003, PASP, 115, 763

Cheung, E., Stark, D. V., Huang, S., et al. 2016, ApJ, 832, 182

Chisholm, J., Tremonti Christy, A., Leitherer, C., \& Chen, Y. 2016, MNRAS, 463, 541

Christensen, C., Quinn, T., Governato, F., et al. 2012, MNRAS, 425, 3058

Christensen, C. R., Davé, R., Governato, F., et al. 2016, ApJ, 824, 57

Christensen, C. R., Governato, F., Quinn, T., et al. 2014, MNRAS, 440, 2843

Cooksey, K. L., Kao, M. M., Simcoe, R. A., O’Meara, J. M., \& Prochaska, J. X. 2013, ApJ, 763, 37

Creasey, P., Theuns, T., \& Bower, R. G. 2015, MNRAS, 446, 2125

Crighton, N. H. M., Hennawi, J. F., \& Prochaska, J. X. 2013, ApJL, 776, L18

Davé, R., Finlator, K., \& Oppenheimer, B. D. 2012, MNRAS, 421, 98

Davé, R., Oppenheimer, B. D., \& Finlator, K. 2011, MNRAS, 415, 11

Dekel, A., \& Mandelker, N. 2014, MNRAS, 444, 2071

D’Odorico, V., Cupani, G., Cristiani, S., et al. 2013, MNRAS, 435, 1198

Erb, D., Shapley, A. E., Pettini, M., et al. 2006, ApJ, 644, 813

Erb, D. K. 2008, ApJ, 674, 151

Faucher-Giguère, C.-A. 2018, MNRAS, 473, 3717

Ferland, G. J., Korista, K., Verner, D. A., et al. 1998, PASP, 110, 761

Finlator, K., \& Davé, R. 2008, MNRAS, 385, 2181

Ford, A. B., Dave, R., Oppenheimer, B. D., et al. 2014, MNRAS, 444, 1260

Ford, A. B., Oppenheimer, B. D., Davé, R., et al. 2013, MNRAS, 432, 89

Gallazzi, A., Charlot, S., Brinchmann, J., White, S. D. M., \& Tremonti, C. A. 2005, MNRAS, 362, 41

Gill, S. P. D., Knebe, A., \& Gibson, B. K. 2004, MNRAS, 351, 399

Governato, F., Zolotov, A., Pontzen, A., et al. 2012, MNRAS, 422, 1231

Haardt, F., \& Madau, P. 1996, ApJ, 461, 20

Heckman, T. M., Armus, L., \& Miley, G. K. 1990, ApJS, 74, 833

Henriques, B. M. B., White, S. D. M., Thomas, P. A., et al. 2013, MNRAS, 431, 3373

Hopkins, P. F., Kereš, D., Oñorbe, J., et al. 2013, MNRAS, 445, 581

Hummels, C. B., Bryan, G. L., Smith, B. D., \& Turk, M. J. 2013, MNRAS, 430,1548

Kacprzak, G. G., Churchill, C. W., Steidel, C. C., Spitler, L. R., \& Holtzman, J. A. 2012, MNRAS, 427, 3029

Katz, N., \& White, S. D. M. 1993, ApJ, 412, 455

Kewley, L. J., \& Ellison, S. L. 2008, ApJ, 681, 1183

Kirby, E. N., Cohen, J. G., Guhathakurta, P., et al. 2013, ApJ, 779, 102

Kirby, E. N., Martin, C. L., \& Finlator, K. 2011, ApJL, 742, L25

Knollmann, S. R. S., \& Knebe, A. 2009, ApJS, 182, 608

Kobulnicky, H. A., \& Kewley, L. J. 2004, ApJ, 617, 240

Kroupa, P., Tout, C., \& Gilmore, G. 1993, MNRAS, 262, 545

Lee, H., Skillman, E. D., Cannon, J. M., et al. 2006, ApJ, 647, 970

Lehner, N., Howk, J. C., \& Wakker, B. P. 2015, ApJ, 804, 79

Lehner, N., O’Meara, J. M., Fox, A. J., et al. 2014, ApJ, 788, 119

Lilly, S. J., Carollo, C. M., Pipino, A., Renzini, A., \& Peng, Y. 2013, ApJ, 772,119

Ma, X., Hopkins, P. F., Faucher-Giguère, C.-A., et al. 2016, MNRAS, 456,2140 
Mannucci, F., Cresci, G., Maiolino, R., et al. 2009, MNRAS, 398, 1915 Martin, C. L. 2005, ApJ, 621, 227

McKee, C. F., \& Ostriker, J. P. 1977, ApJ, 218, 148

McQuinn, K. B. W., Skillman, E. D., Dolphin, A., et al. 2015, ApJL, 815, L17

Michael Shull, J., Danforth, C. W., \& Tilton, E. M. 2014, ApJ, 796, 49

Munshi, F., Governato, F., Brooks, A. M., et al. 2013, ApJ, 766, 56

Muratov, A. L., Kereš, D., Faucher-Giguère, C.-A., et al. 2015, MNRAS, 454, 2691

Muratov, A. L., Kereš, D., Faucher-Giguère, C.-A., et al. 2017, MNRAS, 468, 4170

Nelson, D., Vogelsberger, M., Genel, S., et al. 2013, MNRAS, 429, 3353

Oppenheimer, B. D., \& Davé, R. 2008, MNRAS, 387, 577

Oppenheimer, B. D., Davé, R., Kereš, D., et al. 2010, MNRAS, 406, 2325

Pagel, B. E. J. 1999, arXiv:astro-ph/9911204

Peeples, M. S., \& Shankar, F. 2011, MNRAS, 417, 2962

Peeples, M. S., Werk, J. K., Tumlinson, J., et al. 2014, ApJ, 786, 54

Pettini, M., \& Pagel, B. E. J. 2004, MNRAS, 348, L59

Pettini, M., Shapley, A. E., Steidel, C. C., et al. 2001, ApJ, 554, 981

Pratt, C. T., Stocke, J. T., Keeney, B. A., \& Danforth, C. W. 2018, ApJ, 855, 18

Prochaska, J. X., Weiner, B., Chen, H.-W., Mulchaey, J., \& Cooksey, K. 2011, ApJ, 740, 91

Raiteri, C., Villata, M., \& Navarro, J. F. 1996, A\&A, 315, 105

Rubin, K. H. R., Prochaska, J. X., Koo, D. C., et al. 2014, ApJ, 794, 156

Salim, S., Rich, R. M., Charlot, S., et al. 2007, ApJS, 173, 267

Sanders, R. L., Shapley, A. E., Kriek, M., et al. 2015, ApJ, 799, 138

Santini, P., Fontana, A., Castellano, M., et al. 2017, ApJ, 847, 76

Scannapieco, C., Wadepuhl, M., Parry, O. H., et al. 2012, MNRAS, 423, 1726

Shapley, A. E., Steidel, C. C., Pettini, M., \& Adelberger, K. L. 2003, ApJ, 588, 65

Shen, S., Madau, P., Aguirre, A., et al. 2012, ApJ, 760, 50

Shen, S., Madau, P., Conroy, C., Governato, F., \& Mayer, L. 2014, ApJ, 792, 99
Shen, S., Madau, P., Guedes, J., et al. 2013, ApJ, 765, 89

Shen, S., Wadsley, J., \& Stinson, G. S. 2010, MNRAS, 407, 1581

Smagorinsky, J. 1963, MWRv, 91, 99

Spergel, D. N., Bean, R., Doré, O., et al. 2007, ApJS, 170, 377

Spitoni, E., Calura, F., Matteucci, F., \& Recchi, S. 2010, A\&A, 514, A73

Springel, V., \& Hernquist, L. 2003, MNRAS, 339, 289

Stadel, J. 2001, PhD thesis, Univ. Washington

Steidel, C. C., Erb, D. D. K., Shapley, A. E., et al. 2010, ApJ, 717, 289

Steidel, C. C., Rudie, G. C., Strom, A. L., et al. 2014, ApJ, 795, 165

Stinson, G. S., Brook, C., Maccio, A. V., et al. 2012a, MNRAS, 428, 129

Stinson, G. S., Brook, C., Prochaska, J. X., et al. 2012b, MNRAS, 425, 1270

Stinson, G. S., Seth, A. C., Katz, N., et al. 2006, MNRAS, 373, 1074

Suresh, J., Bird, S., Vogelsberger, M., et al. 2015, MNRAS, 448, 895

Thielemann, F.-K., Nomoto, K., \& Yokoi, K. 1986, A\&A, 158, 17

Torrey, P., Vogelsberger, M., Hernquist, L., et al. 2018, MNRAS, 477, L16

Tremonti, C., Heckman, T. M., Kauffmann, G., et al. 2004, ApJ, 613, 898

Tumlinson, J., Peeples, M. S., \& Werk, J. K. 2017, ARA\&A, 55, 389

Tumlinson, J., Thom, C., Werk, J. K., et al. 2011, Sci, 334, 948

Übler, H., Naab, T., Oser, L., et al. 2014, MNRAS, 443, 2092

Verner, D. A., \& Ferland, G. J. 1996, ApJS, 103, 467

Vogelsberger, M., Genel, S., Springel, V., et al. 2014, MNRAS, 444, 1518

Wadsley, J., Stadel, J., \& Quinn, T. R. 2004, NewA, 9, 137

Weidemann, V. 1987, A\&A, 188, 74

Weiner, B. J., Coil, A. L. A., Prochaska, J. X., et al. 2009, ApJ, 692, 187

Whitaker, K. E., Franx, M., Leja, J., et al. 2014, ApJ, 795, 104

White, C. E., Somerville, R. S., \& Ferguson, H. C. 2015, ApJ, 799, 201

Woods, R. M., Wadsley, J., Couchman, H. M. P., Stinson, G., \& Shen, S. 2014, MNRAS, 442, 732

Woosley, S. E., \& Weaver, T. A. 1995, ApJS, 101, 181

Zahid, H. J., Dima, G. I., Kudritzki, R.-P., et al. 2014, ApJ, 791, 130

Zahid, H. J., Kewley, L. J., \& Bresolin, F. 2011, ApJ, 730, 137

Zolotov, A., Brooks, A. M., Willman, B., et al. 2012, ApJ, 761, 71 